\title{
UMA PROVA ELEMENTAR DA HIPOTESE DE RIEMANN PARA r.URVAS ALGEBRICAS SOBRE CORPOS FINITOS
}

Paulo Agozzini Martin

\author{
DISSERTAÇÃO APRESENTADA AO \\ INSTITUTO DE MATEMATICA E ESTATISTICA \\ DA \\ UNIVERSIDADE DE SATO PAULO \\ PARA OBTENÇÃO DO GRAU DE MESTRE \\ EM \\ MATEMĀT I CA \\ ĀrEA dE CONCENTRAÇĀo: ĀLgebra \\ ORIENTADOR: \\ Prof. Dr. WALTER RICARDO FERRER
}

Durante a elaboração deste trabalho o autor recebeu apoio financeiro da

FAPESP

São Paulo, abril de 1986 


\section{INDICE:}

pag .

INTRODUÇÃO

CAPITULO I - PRELIMINARES ALGEBRICOS ............ 09

$\S 1$ - Corpos finitos ................... 09

$\S 2$ - Valorizações discretas .............. 11

CAPITULO II - INTRODUÇÃO A GEOMETRIA ALGEBRICA ..... 17

$\S 1$ - Variedades afins .................. 17

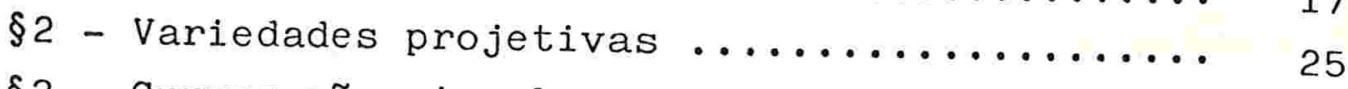

$\S 3$ - Curvas não singulares .............. 33

CAPITULO III - O ENUNCIADO DO TEOREMA DE

RIEMANN-ROCH $\ldots \ldots \ldots \ldots \ldots \ldots \ldots \ldots \ldots \ldots$

CAPITULO IV - A HIPOTESE DE RIEMANN ............ 46

$\S 1$ - A função zeta de uma variedade algébrica .. 46

$\S 2$ - o caso de curvas algébricas ............ 54

$\S 3$ - Prova da hipótese de Riemann para curvas .. 64 


\section{INTRODUCGO}

Um dos problemas centrais da teoria dos números é o da solubilidade de equações diofantinas. E uma das técni cas elementares mais utilizadas no ataque de questões diofan tinas, pela sua simplicidade e eficácia, é a de procurar so luções módulo p. Consideremos, por exemplo, a equação

$$
y^{2}=x^{3}-x-1
$$

onde procuramos encontrar soluções inteiras. Se pensarmos nessa equação módulo 3 , é claro que o lado esquerdo só pode ser 0 ou $1(\bmod 3)$, enquanto o lado direito é $-1(\bmod 3)$, e portanto essa equação não possui soluções inteiras.

Ao estudar as soluções de uma congruência

$$
f\left(x_{1}, x_{2}, \ldots, x_{n}\right) \equiv 0 \quad(\bmod p)
$$

é natural considerar soluções também nas extensões finitas $F_{p} m$ de $F_{p}$. Buscando condensar toda a informação diofantina, Artin, [1], introduziu em 1924 a função zeta de uma varieda de algébrica $X$ sobre um corpo finito $\mathbb{F}_{q}$ :

$$
Z\left(X / F_{q} ; t\right)=\exp \left(\sum_{m \geq 1} N_{m} \frac{t^{m}}{m}\right)
$$

onde $\mathrm{N}_{\mathrm{m}}$ é o número de soluções de $\mathrm{X}$ com coordenadas em ${ }_{\mathrm{F}} \mathrm{m}$. De fato, Artin utilizava a linguagem de corpos de funções al gébricas de uma variável e definiu a função zeta por analo gia com a função zeta de um corpo de números algébricos. E foi também Artin que formulou a "hipótese de Riemann" para a função zeta: os zeros de $\mathrm{Z}\left(\mathrm{X} / \mathrm{F}_{\mathrm{q}} ; \mathrm{q}^{-\mathrm{S}}\right)$ estão todos na reta $\operatorname{Re}(s)=1 / 2$. (Fizemos a mudança de variável $t=q^{-s}$ ). 
Foi somente em 1931 que F.K.Schmidt, [7], mostrou que o teorema de Riemann-Roch podia ser usado para provar que a função zeta de uma curva $x / \mathbb{F}_{q}$ de gênero $g$ é uma função racional de $t=q^{-S}$, da forma:

$$
\zeta(s)=\frac{P\left(q^{-s}\right)}{\left(1-q^{-s}\right)\left(1-q^{1-s}\right)}
$$

onde $P(t)=\underset{i=1}{2 g}\left(1-\alpha_{i} t\right)$ é um polinômio de grau $2 g$ com coefi cientes em $\mathbb{Z}$ e tal que a aplicação $\alpha \rightarrow q / \alpha$ é uma permutação do conjunto das raízes de $P(t)$. Em termos da variável comple $x a s$, isso quer dizer que $P\left(q^{-s}\right)$ satisfaz uma equaça funció nal sob a troca $s+1-s$.

A "hipótese de Riemann" en termos dos $\alpha_{i}$ fica:

$$
\left|\alpha_{i}\right|=q^{1 / 2}
$$

abaixo:

Tomando o logarítmo em ambos os lados da igualdade

$$
\exp \left(\sum_{m \geq 1} N_{m} \frac{t^{m}}{m}\right)=\frac{i_{i=1}^{2 g}\left(1-\alpha_{i} t\right)}{(1-t)(1-q t)}
$$

obtemos:

$$
N_{m}=1+q^{m}-\sum_{i=1}^{2 g} \alpha_{i}^{m}
$$

que é precisamente o número de soluções da curva $X$ na exten são $F_{q} m / F_{q}$.

Assumindo a "hipótese de Riemann" $\left|\alpha_{i}\right|=q^{1 / 2}$, obtemos imediatamente da expressão acima: 


$$
\left|N_{m}-1-q^{m}\right| \leq 2 g q^{m / 2}
$$

E Hase, [5], observou que a desigualdade acima implica, por sua vez a "hipótese de Riemann".

Ou seja, no caso de curvas algébricas, a "hipótese de Riemann" é equivalente a uma desigualdade puramente dio fantina.

o primeiro caso em que a "hipótese de Riemann" foi verificada foi provado por Gauss, [4], para a curva $\mathrm{y}^{2}=\mathrm{x}^{4}-1$. Artin estabeleceu alguns outros casos particula res, e em 1933 Hasse provou o resultado para uma curva elíp tica arbitrária.

Foi somente em 1940 que Weil, [8], provou a "hipó tese de Riemann" para curvas de gênero arbitrário.

Num artigo bem conhecido de Weil, de 1949, ele conjecturou o que deveria ser verdadeiro para variedades de dimensão maior. Seja X uma variedade projetiva não singular de dimensão n sobre $\mathbb{F}_{\mathrm{q}}$. Então:

$$
\begin{aligned}
& \text { (1) } Z\left(X / F_{q} ; t\right) \text { é uma função racional de } t . \\
& \text { (2) } Z\left(X / F_{q} ; t\right)=\frac{P_{1}(t) P_{3}(t) \ldots P_{2 n-1}(t)}{P_{0}(t) P_{2}(t) \ldots P_{2 n}(t)} \text { onde } \\
& P_{i}(t)=\prod_{j=1}^{b}\left(1-\alpha_{i j} t\right), \text { com }\left|\alpha_{i j}\right|=q^{i / 2}
\end{aligned}
$$

(A última igualdade é a "hipótese de Riemann" nesse contex to). 
(3) Sob a aplicação $\alpha+q^{n} / \alpha$ os $\alpha_{i j}$ são levados bijetivamente nos $\alpha_{2 n-i, j}$

(4) No caso de $X$ ser a "redução módulo p" de uma variedade projetiva não singular $\mathbf{X}$ em característica zero, então $b_{i}$ é o i-ésimo número de Betti de $\mathbf{x}$.

Essas são as famosas "conjecturas de Weil", que foram o impulso básico da Geometria Algébrica por mais de 20 anos. A história das tentativas que se fizeram para provar essas "conjecturas" é extremamente interessante, porém nos levaria longe demais. O leitor interessado pode consultar o artigo de Katz, [6], para uma boa resenha. Diremos somente que o análogo da "hipótese de Riemann" para dimensão arbitrá ria foi provado por Deligne, [3], em 1973, e a prova é ex traordinariamente difícil. Mesmo a prova dada por Weil no cá so de curvas de gênero arbitrário é difícil, e é consideradā uma de suas maiores realizações.

Porém em 1973 Bombieri (baseado em trabalhos de Stepanov) deu uma demonstração da "hipótese de Riemann" para curvas que é "elementar", no sentido de que se utiliza somen te do teorema de Riemann-Roch.

A idéia de Stepanov é simples: dada uma curva pro jetiva não singular $\mathbb{C}$ de gênero $g$ sobre $\mathbb{F}_{q}$, procura-se uma função racional $f$ definida em $\mathbf{C}$, não nula, que tem um zero de ordem $\geq m$ em cada um dos pontos $\mathbf{F}_{\mathrm{q}}$-racionais de $\mathbb{C}$, exceto possivelmente num conjunto fixo de $m_{0}$ pontos racionais de $\mathbb{C}$. Decorre então que

$$
m\left(N_{1}-m_{0}\right) \leq \#(z e r o s \text { de } f)=\#(\text { polos de } f)
$$

e portanto 


$$
N_{1} \leq m_{0}+\frac{\#(\text { polos de } f)}{m}
$$

Assim, se construirmos $f$ com poucos polos teremos uma boa limitação para $\mathrm{N}_{1}$.

A presente dissertação objetiva precisamente apre sentar a prova da "hipótese de Riemann" para curvas segun do a versão de Bambieri-Stepanov, [2]. Embora o artigo de Bombieri tenha somente 3,5 folhas e fizéssemos o esforço pa ra dar uma apresentação autocontida, isso não foi possível de vido à natureza complexa do problema e aos muitos pré-requi sitos que ele exige. Minimizamos a utilização de álgebra co mutativa, procurando sempre que possivel o enfoque geométri co, e na parte concernente a geometria algébrica, nos limita mos à definição clássica de variedade algébrica, cuja teoria está concentrada basicamente no mullstellensatz. Não pude mos, porém, evitar o uso de alguns resultados básicos da teo ria das valorizações acerca de extensões de valorizações que não demonstramos no texto, mas que são encontráveis em qual quer livro de números algébricos.

Procuramos dar uma independência relativa aos capí tulos para facilitar a leitura daqueles que não necessitarem de muitos requisitos básicos. 


\section{RIFTERRINCIAS}

[1] - E. Artin. Quadratische Körper en Gebiete der höheren Kongruenzen I, II, in Collected Papers, Springer-Verlag.

[2] - E. Bombieri. Counting points on curves over finite fields. Séminaire Bourbaki 1972/1973 no 430 .

[3] - P. Deligne. La conjecture de Weil I, II, Publ. Math. IHES 43 (1974) 273-307.

[4] - C.F. Gauss. Werke. (a) vol I pp.445-449

(b) vol II pp.67-92.

[5] - H. Hasse. Uber di] Kongruenzetafunctionen, Sitz-ber. d.Preuss. Akad. d. Wiss. 1934.

[6] - N. Katz. An overview of Deligne's proof of the Riemann Hypothesis for varieties over finite fields. Proccedings of Symposia in Purle Math. vol.28, 1976.

[7] - F.K. Schmidt. Analytische Zahlentheorie in Körpern der Characteristik p. Math. Zeit. $33(1931) .1-32$.

[8] - A. Weil. Sur les courbes algébriques e les varietés qui s'en déduisent. Hermann, Paris, 1948. 


\section{CAPITULO I}

\section{PREUUIMIINARESS ALGEBRICOS}

Neste capitulo vamos enunciar os principais resul tados da teoria dos corpos finitos e das valorizações, prin cipalmente para que a exposição que se segue fique o mais au tocontida possível. Como os resultados e teoremas sãu na sua maior parte elementares e podem ser encontrados em qualquer texto introdutório de álgebra (por exemplo: S. Lang, Alge bra), não os demonstraremos aqui.

\section{\$1 - Corpos ifimitos}

Se F é um corpo finito de q elementos, então é cla ro que $F$ tem característica $p>0$, e portanto pode ser con siderado uma extensão de $\mathbb{Z}_{p}$, o corpo dos inteiros módulo $p$. Assim podemos pensar em $F$ como um $Z_{p}$-espaço vetorial e por tanto $q=p^{n}$ para algum inteiro $n \geq 1$. Ou seja, se $F$ é um corpo finito com q elementos então q é uma potência de certo primo p. Consideremos agora o problema inverso, a saber: da do $\mathrm{q}=\mathrm{p}^{\mathrm{n}}$, para um primo $\mathrm{p}$ e um inteiro $\mathrm{n} \geq 1$, achar um cor po finito que tenha exatamente q elementos.

PROPOSIÇAO 1. Se $n$ é um inteiro $\geq 1$ e p um primo, existe um corpo finito com $\mathrm{p}^{\mathrm{n}}$ elementos, univocamente determinado como subcorpo de um fecho algébrico $\bar{z}_{p}$. Além disso esse corpo é o corpo de decomposição do polinômio $x p^{n}-x$ sobre $z_{p}$ e seus elementos são as raízes desse polinômio.

Em geral, denotaremos por $\mathbb{F}_{\mathrm{q}}$ um corpo finito com $\mathrm{q}$ elementos. Se $\mathrm{m}$ e $\mathrm{n}$ são inteiros $\geq 1$ então decorre da pro posição 1 a 
PROPOSIÇAO 2. A inclusão $\mathbb{F}_{\mathrm{q}}{ }^{\mathrm{n} \subset \mathbb{F}_{\mathrm{q}} \mathrm{m}}$ equivale à $\mathrm{n} / \mathrm{m}$ ( $\mathrm{n}$ divide m).

Se k é um corpo finito com q elementos o grupo mul tiplicativo $k^{*}$ de $k$ é comutativo e tem ordem $(q-1)$. Além dis so, é bem conhecida a seguinte proposição:

PROPOSICTO 3. O grupo multiplicativo $k^{*}$ de $\mathrm{k}$ é cíclico de ordem $\mathrm{q}-1$.

Seja $\mathbb{F}_{q}$ um corpo finito com $q=p^{n}$ elementos e con sideremos a aplicação $\phi: \mathbb{F}_{\mathrm{q}}+\mathbb{F}_{\mathrm{q}}$ dada por $\mathrm{x}+\mathrm{x}^{\mathrm{p}}$. Então $\phi \bar{e}$ um automorfismo de $\mathbb{F}_{\mathrm{q}}$, e é chamado automorfismo de Frobenius de $\mathbb{F}_{\mathrm{q}} / \mathbb{F}_{\mathrm{p}}$. Note-se que $\phi$ deixa $\mathbb{F}_{\mathrm{p}}$ fixo, isto é, $\phi(\mathrm{x})=\mathrm{x}$ para todo $x$ de $F_{p}$. Da teoria de Galois decorrem as duas proposi ções abaixo:

PROPOSIÇAO 4. O grupo dos automorfismos de $\mathbb{F}_{\mathrm{q}}$ é cíclico de ordem $n$, gerado pelo automorfismo de Frebenius de $F_{q} / \mathbb{F}_{p}$, $\mathrm{x}+\mathrm{x}^{\mathrm{p}}$.

PROPOSIÇAO 5. Seja $\mathrm{k}$ um corpo finito com q elementos e $\mathrm{K} / \mathrm{k}$ é uma extensão finita de grau m. Então $\mathrm{K} / \mathrm{K}$ é uma extensão de Galois cujo grupo de Galois é ciclico gerado pelo automorfí mo de Frobenius de $\mathrm{K} / \mathrm{k}, \mathrm{x}+\mathrm{x}^{\mathrm{q}}$.

E útil considerar a seguinte caracterização explí cita do fecho algébrico $\overline{\mathbb{F}_{\mathrm{q}}}$ de um corpo finito $\mathbb{F}_{\mathrm{q}}$ com q elé mentos. Consideremos a torre de corpos

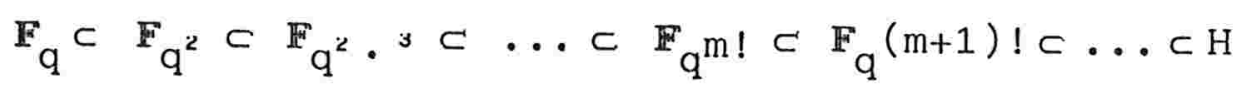

onde $\mathrm{H}=\underset{\mathrm{n} \geq 1}{\mathrm{U}} \mathrm{F}_{\mathrm{q}} \mathrm{n}$ ! E imediata $\mathrm{a}$ : 
PROPOSIÇAO 6. Se $\mathbb{F}_{\mathrm{q}}$ é um corpo finito com q elementos então

$$
\overline{\mathbb{F}_{\mathrm{q}}}=\underset{\mathrm{n} \geq 1}{\mathrm{U}} \mathbb{F}_{\mathrm{q}}^{\mathrm{n}}
$$

e portanto a extensão $\overline{F_{\mathrm{q}}} / \mathbb{F}_{\mathrm{q}}$ é de Galois.

E interessante observar que se $\operatorname{\sigma eGal}\left(\overline{\mathbb{F}}_{\mathrm{q}} / \mathbb{F}_{\mathrm{q}}\right)$ então $\sigma\left(\mathbb{F}_{\mathrm{q}} \mathrm{m} !\right)=\mathbb{F}_{\mathrm{q}} \mathrm{m} !$, ou seja, a restrição de $\sigma$ à $\mathbb{F}_{\mathrm{q}} \mathrm{m}$ pertence à Gal $\left(\mathbb{F}_{q} m ! / \mathbb{F}_{q}\right)$. Essa simples observação será usada no $\S 3$ do Cap. II e no Cap. IV.

\section{$\$ 2$ - Valorizações discretas}

DEFINIÇAO. Seja K um corpo. Uma aplicação v definida no con junto $K^{*}$ dos elementos não nulos de $K$ e tomando valores em $\mathbb{Z}$ diz-se uma valorização discreta de $K$ se:

$$
\begin{aligned}
& \text { (a) } v(a \cdot b)=v(a)+v(b) \\
& \text { (b) } v \text { é sobrejetora } \\
& \text { (c) } v(a+b) \geq \min \{v(a), v(b)\}
\end{aligned}
$$

E cômodo definir $\mathrm{v}(\mathrm{O})=+\infty$ e considerar $\mathrm{v}$ extendi da a K. Vamos reunir num lema as consequências imediatas da definição:

LEMA 1. Seja v uma valorização discreta de um corpo $K$ e sejam $A_{v}=\{\operatorname{aGK}: v(a) \geq 0\}$ e $M_{v}=\{\operatorname{a\in K}: v(a)>0\} \cdot$ Então

$$
\begin{aligned}
& \text { (a) } v(1)=0 ; \quad v\left(a^{-1}\right)=-v(a) \\
& \text { (b) } v(-1)=0 ; \quad v(-a)=v(a) \\
& \text { (c) Se } v(a) \neq v(b) \text { então } a+b \neq 0 \text { e }
\end{aligned}
$$




$$
v(a+b)=\min \{v(a), v(b)\}
$$

(d) $A_{V}$ é um domínio de ideais principais de $\mathrm{K}$ cujo corpo de frações é $K$.

$$
\text { (e) } \mathrm{M}_{\mathrm{V}} \text { é o único ideal primo de } \mathrm{A}_{\mathrm{V}} \text {. }
$$

0 anel $\mathrm{A}_{\mathrm{V}}$ é chama:do o anel da valorização discreta $\mathrm{v}$ e o ideal $\mathrm{M}_{\mathrm{v}}$ é chamado o ideal maximal da valorização $\mathrm{v}$. 0 corpo $\mathrm{A}_{\mathrm{V}} / \mathrm{IM}_{\mathrm{V}}$ e chamado de corpo de restos da valorização dis creta v. Em geral, um anel comutativo com unidade A é chamado de anel de valorização discreta se existir um corpo $\mathrm{K}$ e uma valorização discreta $\mathrm{V}$ de $\mathrm{K}$ tal que $\mathrm{A}=\mathrm{A}_{\mathrm{V}}$. O seguinte lema é uma útil caracterização dos anéis de valorização discreta (para uma prova ver Serre, Corps Locaux):

LEMA 2. Seja A um anel comutativo. Para que A seja um anel de valorizaça discreta é necessário e suficiente que A seja um anel local noetheriano e que seu ideal maximal seja gerado por um elemento não nilpotente.

o próximo lema nos dará uma importante propriedade dos anéis de valorização discreta, que será utilizada adian te:

LEMA 3. Seja K um corpo e v uma valorização discreta de $\mathrm{K}$. Então $A_{V}$ é um subanel maximal de $K$.

Vamos agora enunciar os teoremas mais importantes so bre extensões de valorizações, que serão utilizados nos capí tulos III e IV. Como nesses capítulos estaremos interessados unicamente em corpos de funções algébricas de uma variável, vemos desde já nos situar nesse contexto. 
DEFINIÇAO. Seja k um corpo arbitrário. Um corpo de funções algébricas đe uma variável sobre $\mathrm{k}$ é um corpo $\mathrm{K}$ contendo $\mathrm{k} e$ verificando a condição: $K$ contém um elemento $x$ transcendente sobre $\mathrm{k}$ e $\mathrm{K} / \mathrm{k}(\mathrm{x})$ é uma extensão finita. O fecho algébrico de $\mathrm{k}$ em $\mathrm{K}, \mathrm{k}^{\prime}$, é chamado corpo de constantes de $\mathrm{K}$, e é claro que $\mathrm{K}$ é um corpo de funções algébricas de uma variável sobre $\mathrm{k}^{\prime}$.

DFFINIÇAO. Seja K um corpo de funções algébricas de uma variá vel com corpo de constantes $k$. Uma extensão de $K$ é um corpo de funcõoes algébricas de uma variável L, com corpo de cons tantes 1 tal que $\mathrm{L} \supset \mathrm{K}$ e $\mathrm{I} \cap \mathrm{K}=\mathrm{k}$.

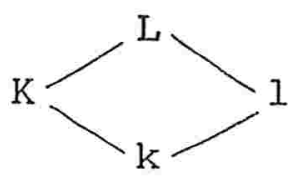

Seja então L/I uma extensão de $\mathrm{K} / \mathrm{k}$ e $\mathrm{w}$ uma valori zação discreta de L trivial sobre 1 . Se $w(x)=0$ para todo xeK w diz-se trivial sobre $K$. Se esse não for o caso, a ima gem $w(K) \subset \mathbb{Z}$ é um subgrupo de $\mathbb{Z}$ da forma ez para certo inteiro positivo $e=e_{L / K}(w)$. E claro que $v=\frac{w}{e_{L / K}(w)}$ é uma valoriza ção discreta de $\mathrm{K}$ trivial sobre $\mathrm{k}$.

0 inteiro $e=e_{L / K}(w)$ é chamado índice de ramifica ção de w sobre $\mathrm{K}$, e a valorização w é dita uma extensão da valorização $\mathrm{v}$.

E fácil ver que $A_{w} \cap K=A_{v}$ e $M_{w} \cap K=M_{v}$, e assim existe uma injeção canônica:

$$
\mathrm{K}_{\mathrm{v}}=\mathrm{A}_{\mathrm{v}} / \mathrm{M}_{\mathrm{v}}+\mathrm{A}_{\mathrm{w}} / \mathrm{M}_{\mathrm{w}}=\mathrm{L}_{\mathrm{w}}
$$

e vale o seguinte lema: 
LEMA 4. Seja L/1 uma extensao de K/K. Então são equivalentes:

(a) $[1: k]<\infty$

(b) $[\mathrm{L}: \mathrm{K}]<\infty$

(c) $\left[\mathrm{L}_{\mathrm{w}}: \mathrm{K}_{\mathrm{V}}\right]<\infty$

Para uma prova ver Deuring, Lectures on the Theory of Algebraic Functions of One Variable, pg 93. 0 inteiro $\left[\mathrm{L}_{\mathrm{W}}: \mathrm{K}_{\mathrm{V}}\right]=\mathrm{d}_{\mathrm{L} / \mathrm{K}}(\mathrm{w})$ é chamado $\mathrm{o}$ grau de $\mathrm{w}$ sobre $\mathrm{K}$.

PROPOSIÇAO 1. Seja $\mathrm{K} / \mathrm{K}$ um corpo de funções algébricas sobre - corpo de constantes $\mathrm{k}$, e $\mathrm{v}$ uma valorização discreta de $\mathrm{K}$, trivial sobre k. Então $\left[\mathrm{K}_{\mathrm{V}}: \mathrm{k}\right]<\infty$. (Ver Deuring, Idem pg 15).

Se L/l é uma extensão de $\mathrm{K} / \mathrm{k}$, é fácil ver que as condições abaixo são equivalentes:

(a') $1 / k$ é algébrica

(b') L/K é algébrica

(c' $) L_{w} / K_{w}$ é algébrica

Assim, se $\mathrm{L} / \mathrm{l}$ extende $\mathrm{K} / \mathrm{k}$, diremos que se trata de uma extensão algébrica se for verificado um dos três itens acima, e diremos que é uma extensão finita se se verifi car um dos itens do lema 4.

E importante observar que se L/l é uma extensão al gébrica de $\mathrm{K} / \mathrm{k}$, então não existe nenhuma valorização discreta $W$ de $L$ que seja trivial sobre $K$. Pois se $w$ fosse trivial em $K$, seja al, e seja $f(x)$ eL[x] o polinômio irredutível de $\alpha$ sobre $\mathrm{K}$ : 


$$
f(\alpha)=\alpha^{n}+a_{1} \alpha^{n-1}+\cdots+a_{n}=0, a_{i} \in K
$$

Então

$$
0=w\left(a_{n}\right)=w\left(\alpha^{n}+a_{1} \alpha^{n-1}+\cdots+a_{n-1}^{\alpha}\right)
$$

Se $w(\alpha)>0$, pelo item (c) do lema 1 deste paragrá fo, então

$$
\left.w\left(\alpha^{n}+a_{1} \alpha^{n-1}+\ldots+a_{n-1} \alpha\right)=\min \left\{w\left(\alpha^{n}\right), \ldots, w(\alpha)\right\}=w^{\prime} \alpha\right)
$$

donde obtemos uma contradição.

TEOREMA 1. Seja L/l uma extensão qualquer de $\mathrm{k} / \mathrm{k}$. Se $\mathrm{v}$ é uma valorização discreta de $\mathrm{K}$, trivial sobre $\mathrm{k}$, o número de todas as possíveis extensões de $v$ à valorizações discretas de L, triviais sobre 1 , é finito e positivo. (Ver Deuring, Idem, pg 96).

No teorema 2 abaixo vamos considerar o caso impor tante de extensões de Galois.

TEOREMA 2. Seja L/l uma extensão de Galois de K/K e v uma valorização discreta de $K$, trivial sobre k. Seja w uma ex tensão de $\mathrm{v}$ à L. Então qualquer extensão de $\mathrm{v}$ à $\mathrm{L}$ estará no conjunto $\left\{w_{o p}: \operatorname{pegal}(\mathrm{L} / \mathrm{K})\right\}$.

Para uma prova desse teorema no caso de extensões L/K finitas ver Deuring, Idem, pg 101. Para o caso geral ver O. Endler, Valuation Theory, pg 109.

TEOREMA 3. Seja L/l uma extensão finita de K/k. Seja v uma valorização discreta de $K$ trivial em $k$, e sejam $w_{1}, \ldots, w_{h}$ todas as extensões de v a L. Então

$$
[L: K]=\sum_{i=1}^{h} d_{L / K}\left(w_{i}\right) e_{L / K}\left(w_{i}\right)
$$


Se, além disso a extensão for de Galois, então $d_{L / K}\left(w_{1}\right)=\ldots=d_{L / K}\left(w_{h}\right)=d e e_{L / K}\left(w_{1}\right)=\ldots=e_{L / K}\left(w_{h}\right)$ $=\mathrm{e}$ e portanto

$$
[\mathrm{L}: \mathrm{K}]=\mathrm{h} \mathrm{d} \mathrm{e}
$$

Para uma demonstração ver Iyanaga, Theory of Numbers, pg 1.38 .

o lema seguinte é extremamente importante para o que se segue:

LEMA 5. Se L/l é una extensão algébrica separável de $\mathrm{K} / \mathrm{K}$ e tal que $L=K I$ então toda valorização discreta $w$ de $L$, trí vial sobre $l$, tem índice de ramificação $e_{L / K}(w)=1$, sobre K. (Ver Deuring, pg. 113).

Assim a restrição $\mathrm{w} / \mathrm{k}$ será uma valorização discre ta de $\mathrm{K}$ trivial sobre $\mathrm{k}$. 


\section{CAPPITUILO III}

\section{IIN"RODUGGO A GEONETIRTA ALGEBRTCA}

\section{\$1 - Variedades afins}

A geometria algébrica clássica trata do estudo geo métrico do conjunto das soluções de equações polinomiais so bre o corpo dos números complexos. Em geral, porém, estamos interessados nas soluções de equações polinomiais com coefi cientes num corpo arbitrário $\mathrm{k}$, eventualmente de caracterís tica $\mathrm{p}>0$. Isso nos leva a introduzir o conceito de $\mathrm{K} / \mathrm{k}$ variedade, que analisaremos a seguir. No que se segue $k$ é um corpo arbitrário, $\mathrm{K} / \mathrm{K}$ uma extensão de corpos com $\mathrm{K}$ alge bricamente fechado.

Indicaremos por $\mathbb{A}_{K}^{n}$ o conjunto das n-uplas de ele mentos de $K$, o n-espaço afim sobre $K$.

Seja $J$ um subconjunto arbitrário do anel de poli nômios $\mathrm{k}\left[\mathrm{x}_{1}, \mathrm{x}_{2}, \ldots, \mathrm{x}_{\mathrm{n}}\right]$. Definimos:

$$
V_{K}(J)=\left\{\left(a_{1}, \ldots, a_{n} \in \mathbb{A}_{K}^{n}: f\left(a_{1}, \ldots, a_{n}\right)=0, \quad \forall f e J\right\}\right.
$$

Da definição acima decorre que se $A$ e $B$ são subcon juntos de $k\left[X_{1}, x_{2}, \ldots, x_{n}\right] \operatorname{com} A \subset B$ então $V_{K}(A) \supset V_{K}(B)$. Se $\mathrm{J}$ é um subconjunto não vazio de $\mathrm{k}\left[\mathrm{X}_{1}, \mathrm{X}_{2}, \ldots, \mathrm{X}_{\mathrm{n}}\right]$ denotamos por $\langle\mathrm{J}\rangle$ o ideal gerado por $\mathrm{J}$ e por $\operatorname{Rad}(\langle\mathrm{J}\rangle)$ o radical do ideal $<\mathrm{J}>$.

E fácil ver que valem as igualdades:

$$
\mathrm{V}_{\mathrm{K}}(\mathrm{J})=\mathrm{V}_{\mathrm{K}}(\langle\mathrm{J}\rangle)=\mathrm{V}_{\mathrm{K}}(\operatorname{Rad}(\langle\mathrm{J}\rangle))
$$


ideais de $\mathrm{k}\left[\mathrm{X}_{1}, \mathrm{X}_{2}, \ldots, \mathrm{x}_{\mathrm{n}}\right]$ em vez de subconjuntos arbitrá rios.

Se $J_{1}$ e $J_{2}$ são ideais de $k\left[x_{1}, x_{2}, \ldots, x_{n}\right], J_{1} J_{2}$ designa o ideal gerado pelo conjunto $\left\{a b: a \in J_{1}, b \in J_{2}\right\}$. E cla ro que:

$$
V_{K}\left(J_{1} J_{2}\right)=V_{K}\left(J_{1}\right) \cup V_{K}\left(J_{2}\right)
$$

e se $\left\{J_{\alpha}\right\}$ é uma família de ideais de $k\left[X_{1}, X_{2}, \ldots, X_{n}\right]$ en tão:

$$
\mathrm{V}_{\mathrm{K}}\left(\underset{\alpha}{\mathrm{U}} \mathrm{J}_{\alpha}>\right)=\underset{\alpha}{\bigcap} \mathrm{V}_{\mathrm{K}}\left(\mathrm{J}_{\alpha}\right)
$$

Vamos agora definir uma topologia em $\mathbb{A}_{K}^{n}$ tomando os subconjuntos $V_{K}(J)$ como os fechados de $\mathbb{A}_{K}^{n}$, para $J$ per correndo os ideais de $\mathrm{k}\left[\mathrm{x}_{1}, \mathrm{x}_{2}, \ldots, \mathrm{x}_{\mathrm{n}}\right]$.

$$
\text { Note-se que } \mathbb{A}_{K}^{n} \text { e } \varnothing \text { são fechados e que, por }
$$

a intersecção de uma família qualquer de fechados é um fecha do, e por (2), a união de dois fechados é um fechado, de mo do que temos efetivamente definida uma topologia em $\mathbb{A}_{K}^{n}$, cha mada a k-topologia de Zariski de $\mathbb{A}_{K}^{n}$.

DEFINIÇAO. Uma $\mathrm{K} / \mathrm{k}$-variedade afim é um subconjunto fechado de $\mathbb{A}_{K}^{n}$ na k-topologia de Zariski.

Seja $\mathrm{S} \subset \mathbb{A}_{K}^{\mathrm{n}}$ um subconjunto arbitrário. Definimos o ideal de $\mathrm{S}$ em $\mathrm{k}\left[\mathrm{x}_{1}, \mathrm{x}_{2}, \ldots, \mathrm{x}_{\mathrm{n}}\right]$ por:

$$
I_{k}(S)=\left\{\operatorname{fek}\left[X_{1}, X_{2}, \ldots, X_{n}\right]: f(P)=0, \quad \text { vPes }\right\}
$$


onde $P$ representa uma $n$-upla $\left(a_{1}, \ldots, a_{n}\right)$ es.

$$
\text { E claro que se } k \subset k_{1} \subset K \quad \text { e } S \subset \mathbb{A}_{K}^{n} \text {, então: }
$$

$$
I_{k_{1}}(S) \supset I_{k}(S)
$$

$$
I_{k_{1}}(S) \cap k\left[X_{1}, X_{2}, \ldots, X_{n}\right]=I_{k}(S)
$$

Se $S_{1}$ e $S_{2}$ são sunconjuntos não vazios de $\mathbb{A}_{K}^{n}$ en tão $I_{k}\left(S_{1} \cup S_{2}\right)=I_{k}\left(S_{1}\right) \cap I_{k}\left(S_{2}\right) ; \quad$ se $S_{1} \subset S_{2}$ então $I_{k}\left(S_{1}\right) \supset I_{k}\left(S_{2}\right)$. Se $J$ é um ideal de $k\left[X_{1}, x_{2}, \ldots, X_{n}\right]$ e $S \subset \mathbb{A}_{K}^{\mathrm{n}}$ temos:

$$
\mathrm{V}_{\mathrm{K}}\left(\mathrm{I}_{\mathrm{K}}(\mathrm{S})\right)>\mathrm{S}
$$

(7)

$$
I_{k}\left(V_{K}(J)\right) \supset J
$$

Aplicando $I_{k}$ à primeira expressão de (7) obtemos $I_{k}\left(V_{K}\left(I_{k}(S)\right)\right) \subset I_{k}(S)$. Como $I_{k}(S)$ é um ideal, a segunda ex pressão de (7) nos dá: $I_{k}\left(V_{K}\left(I_{k}(S)\right)\right) \supset I_{k}(S)$, donde temos:

$$
I_{k}\left(V_{K}\left(I_{k}(S)\right)\right)=I_{k}(S)
$$

\section{Analogamente obtemos}

$$
\mathrm{V}_{\mathrm{K}}\left(\mathrm{I}_{\mathrm{k}}\left(\mathrm{V}_{\mathrm{K}}(\mathrm{J})\right)\right)=\mathrm{V}_{\mathrm{K}}(\mathrm{J})
$$

Observe-se que se $\mathrm{X} \subset \mathbb{A}_{\mathrm{K}}^{\mathrm{n}}$ é um fechado na k-topolo gia de $\mathbb{A}_{K}^{n}$ então, por $(9), V_{K}\left(I_{k}(X)\right)=x$.

Vimos que $I_{k}\left(V_{K}(J)\right) \supset J$. Além disso, é claro que $I_{k}\left(V_{K}(J)\right) \supset \operatorname{Rad}(J)$. No caso particular em que $k=K$ temos 
$I_{K}\left(V_{K}(J)\right)=\operatorname{Rad}(J)$. Esse é o conteúdo do célebre:

NULLSTELLENSATZ (Hilbert). Seja $\mathrm{K}$ um corpo algebricamente fechado e $J$ um ideal de $K\left[x_{1}, x_{2}, \ldots x_{n}\right]$. Se $f$ é um polinộ mio de $\mathrm{K}\left[\mathrm{X}_{1}, \mathrm{X}_{2}, \ldots, \mathrm{X}_{\mathrm{n}}\right]$ que se anula em todos os pontos de $V_{K}(J)$ então $\operatorname{feRad}(J)$.

Para uma demonstração bastante curta desse teorema ver a nota de Artin-Tate, [1], "A note on finite ring extensions". No caso em que $k_{q} \mathrm{~K}$ temos a versão:

PROPOSICAO 1. Seja $J$ um ideal de $k\left[x_{1}, x_{2}, \ldots, x_{n}\right], k / k$ uma extensão de corpos com $K$ algebricamente fechado. Então $I_{k}\left(V_{K}(J)\right)=\operatorname{Rad}(J)$.

PROVA. Vamos denotar por $\mathrm{J} \otimes \mathrm{K} \circ$ ideal de $\mathrm{K}\left[\mathrm{X}_{1}, \mathrm{X}_{2}, \ldots, \mathrm{X}_{\mathrm{n}}\right]$ gerado por $\mathrm{J}$, isto é, o ideal extendido. E claro que $V_{K}(J \otimes K)=V_{K}(J)$. Do nullstellensatz temos: $I_{K}\left(V_{K}(J \otimes K)\right)=$ $\operatorname{Rad}(\mathrm{J} \otimes \mathrm{K})$.

Donde segue:

$$
\begin{aligned}
& I_{K}\left(V_{K}(J \otimes K)\right) \cap k\left[X_{1}, X_{2}, \ldots, X_{n}\right]= \\
& \quad=\operatorname{Rad}(J \otimes K) \cap k\left[X_{1}, X_{2}, \ldots, X_{n}\right]
\end{aligned}
$$

e por (6),

$$
\begin{aligned}
& I_{k}\left(V_{K}(J \otimes K)\right)=I_{k}\left(V_{K}(J)\right)= \\
& =\operatorname{Rad}(J \otimes K) \cap k\left[X_{1}, X_{2}, \ldots, X_{n}\right]
\end{aligned}
$$


Resta provar que a contração do radical da exten são do ideal $J$ é o próprio $\operatorname{Rad}(J)$. Isso é um resultado sim ples de álgebra comutativa que pode ser visto, por exemplo, em [2], pg 10.

OBSERVAÇAO 1. Se K é um corpo algebricamente fechado, decor re imediatamente do nullstellensatz a existência de uma cor respondência biunívoca entre os fechados de $\mathbb{A}_{K}^{n}$ e os ideais radicais (isto é, os ideais que são iguais aos seus radi cais), dada por $S+I_{K}(S)$ e $J+V_{K}(J)$.

OBSERVAÇAO 2. Vimos anteriormente que se $X$ for um fechado de $\mathbb{A}_{K}^{n}$ então $V_{K}\left(I_{K}(X)\right)=X$. Se $Y$ é um subconjunto qualquer de $\mathbb{A}_{K}^{n}$, então o seu fecho $\bar{Y}$ na k-topologia de Zariski é $V_{K}\left(I_{K}(Y)\right.$ ) pois se $V$ é um fechado que contém $Y$, então $I_{k}(V) \subset I_{k}(Y)$ e $\operatorname{assim} V=V_{K}\left(I_{K}(V)\right) \supset V_{K}\left(I_{k}(Y)\right)$, donde $V_{k}\left(I_{k}(Y)\right)$ é o menor $f \underline{e}$ chado que contém $Y$.

DEFINIÇశO. Um espaço topológico X diz-se noetheriano se os seus subconjuntos abertos satisfazem a condição de cadeia ascendente.

DEFINIÇAO. Um espaço topológico $\mathrm{x}$ é dito irredutível se sạ tisfizer uma das três condições equivalentes:

(a) X não é a união de dois fechados prórpios de $\mathrm{X}$.

(b) A intersecção de dois abertos próprios de X é não vazia.

(c) Todo aberto não vazio é denso.

Observe-se que se $X$ é um espaço topológico irredutí vel e ACX for um aberto não vazio, então A será um espaço 
topológico irredutível, na topologia induzida.

Suponhamos que $V \subset \mathbb{A}_{K}^{n}$ seja uma $K / k$-variedade afim. E claro que a cada subconjunto fechado de $\mathrm{V}$ (na topologia in duzida) corresponde um ideal de $\mathrm{k}\left[\mathrm{X}_{1}, \mathrm{x}_{2}, \ldots \mathrm{x}_{\mathrm{n}}\right]$ que contém $I_{k}(V)$ pois os fechados de $V$ na topologia induzida são os fe chados de $\mathbb{A}_{K}^{n}$ contidos em $V$. Reciprocamente, a cada ideal de $k\left[X_{1}, X_{2}, \ldots, X_{n}\right]$ que contém $I_{k}(\dot{V})$ corresponde um subcon junto fechado de $\mathrm{V}$.

$E$ bem conhecido que os ideais de $\mathrm{k}\left[\mathrm{X}_{1}, \mathrm{x}_{2}, \ldots, \mathrm{x}_{\mathrm{n}}\right]$ que contém $I_{k}(V)$ estão em correspondência bijetora com os ideais de $k\left[x_{1}, x_{2}, \ldots, x_{n}\right] / I_{k}(V)$. No que se segue denotare mos esse quocjente por k[V]. Esse anel é chamado de anel de coordenadas afins ou anel das funções polinomiais em $v$. Co mo $k\left[X_{1}, \ldots, X_{n}\right]$ é um anel noetheriano, então $k[V]$ será noetheriano, e portanto toda cadeia ascendente de ideais de $\mathrm{k}\left[\mathrm{X}_{1}, \ldots, \mathrm{X}_{\mathrm{n}}\right]$ que contém $\mathrm{I}_{\mathrm{k}}(\mathrm{V})$ estaciona, ou seja, toda ca deia descendente de fechados de $\mathrm{V}$ é estacionária, e portanto $\mathrm{V}$ é um espaço topológico noetheriano com a k-topologia de Zariski induzida.

PROPOSIÇAO 2. A K/k-variedade afim V com a k-topologia induzi da é um espaço topológico irredutível se e só se $I_{k}(V)$ for um ideal primo.

PROVA. Suponhamos que $V$ seja irredutível e que $f_{1}, f_{2}$ sejam dois polinômios fora de $I_{k}(V)$. Seja $W_{i}$ o conjunto dos pontos de $V$ onde $f_{i}$ se anula, $i=1,2$. Então $W_{i}$ é uma $K / k$-variedade de $\mathbb{A}_{K}^{n}$ e também um fechado próprio de $V$. Como $V$ é irredutí vel, $W_{1} U W_{2} \mp V$. Seja $x$ um ponto de $V-\left(W_{1} \cup W_{2}\right)$. Então $f_{i}(x) \neq 0, i=1,2$, e portanto $f_{1} \cdot f_{2} \notin I_{k}(V)$, donde $I_{k}(V)$ é um ideal primo. Reciprocamente, se $I_{k}(V)$ for um ideal pri mo, seja $V=V_{1} \cup V_{2}$, onde $V_{i}$ é um fechado de $V, i=1,2$. 
Suponhamos que $V_{2} \neq V$. Provaremos que $V_{1}=V$ e que, portanto, $\mathrm{V} \dot{e}$ irredutivel. Temos:

$$
I_{k}(V)=I_{k}\left(V_{1} \cup V_{2}\right)=I_{k}\left(V_{1}\right) \cap I_{k}\left(V_{2}\right) \supset I_{k}\left(V_{1}\right) \cdot I_{k}\left(V_{2}\right)
$$

como $I_{k}(V)$ é primo e $I_{k}\left(V_{2}\right) \supsetneq I_{k}(V)$ segue que $I_{k}(V) \supset I_{k}\left(V_{1}\right)$, ou seja $V=V_{1}$.

PROPOSTÇAO 3. Num espaço topológico noetheriano X todo fecha do não vazio $F$ pode ser expresso como a união finita $F=F_{1}$ $U \ldots \cup F_{n}$ de fechados irredutíveis de $X$. Se eliminarmos as redundâncias, isto é, se $F_{i} \not F_{j}$ para $i \neq j$, então a decom posição é única.

PROVA. Seja $\mathbb{F}$ o conjunto dos fechados não vazios de X que não podem ser expressos como uma reunião finita de fechados irre dutíveis. Suponhamos que $\mathbb{F}$ seja não vazio. Como X é noetheriano $F$ possui um elemento minimal $Y$. Logo $Y$ não pode ser irredutí vel e portanto $Y=Y_{1} U Y_{2}$ onde $Y_{i}$ é um fechado próprio de $Y, i=1,2$. Pela minimalidade de $Y, Y_{i} \notin \mathbb{F}$, donde temos uma contradiçäo, e portanto $\mathbb{F}=\varnothing$.

Se $F=F_{1} U F_{2} \cup \ldots U F_{n}$, com $F_{i}$ fechado e irre dutível e se $F=Y_{1} \cup \ldots U Y_{m} \operatorname{com} Y_{i}$ fechado e irredutível, $\mathrm{Y}_{i} \not \mathrm{Y}_{j \mathrm{n}} \mathrm{se} i \neq j, \quad$ como $\quad \mathrm{Y}_{1} \subset \mathrm{F}_{1} \cup \ldots \cup \mathrm{F}_{\mathrm{n}}=\mathrm{F}$, $Y_{1}=i_{i=1}\left(F_{i} \cap Y_{1}\right)$ e como $Y_{1}$ é irredutível, $Y_{1} \subset F_{i(1)} \cdot R \underline{e}$ petindo $\circ$ argumento para $F_{i(1)}$, temos $F_{i(1)} \subset Y_{j}$ para algum índice $j$. Como por hipótese $Y_{i} \not \subset Y_{j}$ se $i \neq j$, segue que $j=1$, ou seja, $Y=F_{i(1)}$, donde $\mathrm{m} \leq \mathrm{n}$. Invertendo $\circ$ argumen

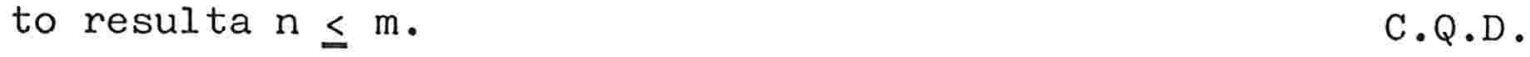

COROLÁRIO 1. TOda K/K-variedade afim V pode ser escrita como uma reunião finita de $\mathrm{K} / \mathrm{k}$-variedades afins irredutíveis $v_{i}, \operatorname{com} v_{i} \not p v_{j}$ se $i \neq j$. 
DEFINIÇO. Se $X$ é um espaço topológico, definimos a dimensão de $X$ (denotada dimX) como o supremo de todos os inteiros $n$ tais que existe uma cadeia $F_{0} \subset F_{1} \subset \ldots \subset F_{n}$ de fechados ir redutíveis e distintos de $X$. Definimos a dimensão de uma $\mathrm{K} / \mathrm{k}$-variedade afim como a sua dimensão como espaço topológi co.

OBSERVAÇAO. Em vista do co:olário $1, \operatorname{dim} V=\max _{i} \operatorname{dim} V_{i}$ onde as $V_{i}$ são as componentes irredutíveis de $V$.

DEFINIÇÃO. Num anel A, a altura de um ideal primo $P$ é o su premo de todos os inteiros $n$ para os quais existe uma cadeia $\mathrm{P}_{0} \subset \mathrm{P}_{1} \subset \ldots \subset \mathrm{P}_{\mathrm{n}}=\mathrm{P}$ de ideais primos distintos. Define-se a dimensão de Krull de A, denotada dimA, como o supremo das alturas de todos os ideais primos de A.

PROPOSIÇAO 4. Se Y é uma K/K-variedade afim então a dimensão de $Y$ é igual à dimensão de Krull de $k[Y]$.

PROVA. Já vimos que os fechados irredutíveis de $Y$ correspon dem a ideais primos de $k\left[X_{1}, X_{2}, \ldots X_{n}\right]$ que contém $I_{k}(Y)$. Estes por sua vez correspondem a ideais primos de $k[Y]$ e re ciprocamente. Assim a dimensão de $Y$ é o comprimento da mais longa cadeia de ideais primos em k[Y], que é sua dimensão de Krull. C.Q.D.

Se $Y$ é uma $K / k$-variedade afim irredutível a propo sição 2 garante que $o$ anel de coordenadas afins $k[Y]$ é um domínio. o corpo de frações de $k[Y]$, denotado $k(Y)$, é chamado o corpo das funções racionais em $\mathrm{Y}$. Como $\mathrm{k}[\mathrm{Y}]$ é um domínio finitamente gerado como k-álgebra, um resultado de álgebra comutativa nos garante que a dimensão de Krull de $k[Y]$ (e portanto a dimensão de $Y$ ) é igual ao grau de transcendência da extensão $\mathrm{k}(\mathrm{Y}) / \mathrm{k}$ (Matsumura, Commutative Algebra, cap 5 , § 14). 
1 é chamada curva afim.

Seja $V$ uma $K / k$ variedade afim irredutível e $f$ uma função racional em V (isto é, fek(V)). Se $P$ é um ponto de $V$, dizemos que $f$ está definida em $P$ se para certos $h$, gヒk[V], $\mathrm{f}=\mathrm{h} / \mathrm{g}$ e $\mathrm{g}(\mathrm{P}) \neq 0$. Definimos $\mathrm{O}_{\mathrm{P}}(\mathrm{V})$ como o conjunto das funções racionais em $V$ que estão definidas em $P$. E imediato verificar que $O_{P}(V)$ é isomarfo ao localizado de $k[V]$ em rela ção ao ideal das funções polinomiais de k[V] que se anulam em P. Assim $O_{P}(V)$ é um subanel de $k(V)$ que contém $k[V]$. o seu único ideal maximal é $\mathrm{M}_{\mathrm{P}}=\left\{\mathrm{feO}_{\mathrm{P}}(\mathrm{V}): \mathrm{f}(\mathrm{P})=0\right\}$. Em particu lar se $k=K$ for algebricamente fechado, $O_{P}(V) / M_{P} \simeq K$.

\section{$\$ 2$ - Variedades projetivas}

De modo análogo ao caso afim, começamos por defi nir o n-espaço projetivo sobre um corpo arbitrário $k$ como o conjunto das $(n+1)$-uplas $\left(a_{0}, a_{1}, \ldots, a_{n}\right)$ de elementos não todos nulos de $\mathrm{k}$ sob a relação de equivalência dada por $\left(a_{0}, a_{1}, \ldots, a_{n}\right) \sim\left(\lambda a_{0}, \lambda a_{1}, \ldots, \lambda a_{n}\right)$ para todo $\lambda \in k$, $\lambda \neq 0$. Denotaremos por $\mathbb{P}_{k}^{n} \circ$ n-espaço projetivo sobre $k$. Um elemento de $\mathbb{P}_{k}^{n}$ é chamado ponto. Se $\mathrm{P}$ é um ponto, uma $(n+1)$ upla $\left(a_{0}, a_{1}, \ldots, a_{n}\right)$ na classe de equivalência $P$ é chamada um conjunto de coordenadas homogêneas para $P$. Observe-se que embora não possamos falar na i-ésima coordenada de $P, a_{i}$ po demos falar que $a_{i}=0$ ou que $a_{i} \neq 0$, e assim definimos 0 conjunto $U_{i}=\left\{\left(x_{0}, x_{1}, \ldots, x_{n}\right) \in \mathbb{P}_{k}^{n}: x_{i} \neq 0\right\}$. Se $P_{i}$, $P=\left(x_{0}, x_{1}, \ldots, x_{n}\right)$, podemos associar à $P$ a n-upla $\left(x_{0} / x_{i}, x_{1} / x_{i}, \ldots, x_{n} / x_{i}\right)$ com $x_{i} / x_{i}$ omitido. Note-se que o quociente $x_{j} / x_{i}$ independe da particular representação de P. Temos então uma aplicação $\phi_{i}: U_{i}+\mathbb{A}_{k}^{n}$ que estabelece uma correspondência bijetora entre os pontos de $U_{i}$ os pon 
tos de $\mathbb{A}_{k}^{n}$. Além disso $\mathbb{P}_{k}^{n}={ }_{i=1}^{n} U_{i}$.

Se $f \in k\left[x_{0}, x_{1}, \ldots, x_{n}\right]$ é um polinômio, não pode mos utilizá-lo para definir uma função em $\mathbb{P}^{n}$, pela não uni cidade das coordenadas homogêneas. Entretanto, se f for um polinômio homogêneo de grau $d$, isto é, se $f\left(\lambda a_{0}, \ldots, \lambda a_{n}\right)=$

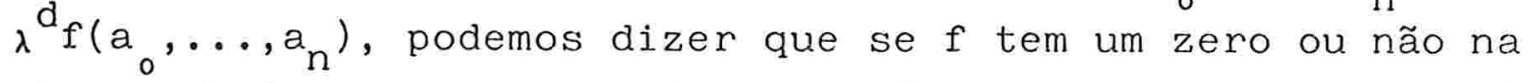
classe de $\left(a_{0}, a_{1}, \ldots, a_{n}\right)$. Se $J$ é um conjunto de polinô mios homogêneos de $k\left[x_{0}, x_{1}, \ldots, x_{n}\right]$ e $k / k$ uma extensão de corpos, definimos:

$$
V_{K}(J)=\left\{P \in \mathbb{P}_{k}^{n}: f(P)=0 \quad \forall f \in J\right\}
$$

Dizemos que um ideal de $\mathrm{k}\left[\mathrm{x}_{0}, \mathrm{x}_{1}, \ldots, \mathrm{x}_{\mathrm{n}}\right]$ é homo gêneo se for gerado por elementos homogêneos. Como no caso afim, se $\mathrm{J}$ é como acima, vale $\mathrm{V}_{\mathrm{K}}(\mathrm{J})=\mathrm{V}_{\mathrm{K}}(\langle\mathrm{J}\rangle)$ e portanto pas saremos a considerar somente ideais homogêneos de $\mathrm{k}\left[\mathrm{x}_{0}, \ldots, \mathrm{x}_{\mathrm{n}}\right]$.

Definimos a k-topologia de Zariski de $\mathbb{P}_{K}^{n}$ de modo análogo ao caso afim, tomando os subconjuntos $V_{K}(J)$ como os fechados, onde $\mathrm{J}$ percorre o conjunto dos ideais homogêneos de $k\left[x_{0}, x_{1}, \ldots x_{n}\right]$. A verificação de que de fato temos uma topologia é análoga ao caso afim. Obtida a topologia, as noções de subconjunto irredutível e de dimensão se aplicam naturalmente.

DEFINIÇAO . Uma $\mathrm{K} / \mathrm{k}$-variedade projetiva é um subconjunto fechado de $\mathbb{P}_{K}^{n}$, com a topologia induzida. A dimensão de uma variedade projetiva é a sua dimensão como espaço topológico.

Se $S$ é um subconjunto não vazio de $\mathbb{P}_{K}^{n}$, definimos $I_{k}(S)$ como o ideal gerado pelo conjunto $\left\{f G k\left[x_{0}, X_{1}, \ldots, X_{n}\right]\right.$, 
homogêneo, : $f(P)=0, \forall P \in S\}$, e portanto é um ideal homogê neo. Se I é um ideal homogêneo, I é um ideal primo se e só se para dois quaisquer polinômios homogêneos $f$ e $g$, se f.geI então ou feI ou geI. Com esse resultado, podemos repe tir quase inteiramente a prova dada no caso afim e concluir que $V$ é uma $K / K$-variedade projetiva irredutível se e só se $I_{k}(V)$ for um ideal primo. Assim o anel $k_{h}[V]=k\left[X_{0}, X_{1}, \ldots\right.$, $\mathrm{X}_{\mathrm{n}}$ !/I $\mathrm{I}_{\mathrm{k}}(\mathrm{V})$ é um domínio e é chamado anel de coordenadas homo gêneas de $V$. O corpo de frações de $k_{h}[V]$, denotado $k_{h}(V)$, é chamado o corpo de funções homogêneas de $V$.

Ao contrário do caso afim, os elementos de $k_{h}(V)$ em geral não determinam funções em $V$. No que se segue vere mos como construir de fato um corpo de funções em $V$.

$\underline{\text { PROPOSICAO } 1}$ i Seja $I \subset k\left[X_{0}, X_{1}, \ldots, X_{n}\right]$ um ideal homogê neo. Se $F=\sum_{i=0} F_{i}$ é um elemento de $I$, onde $F_{i}$ é um polinômio homogêneo de grau $i$, então tem-se que $F_{i} \in I, i=0,1, \ldots, m$.

PROVA. Basta provar que $F_{m} G I$, pois então $F-F_{m} E I$ e o resto

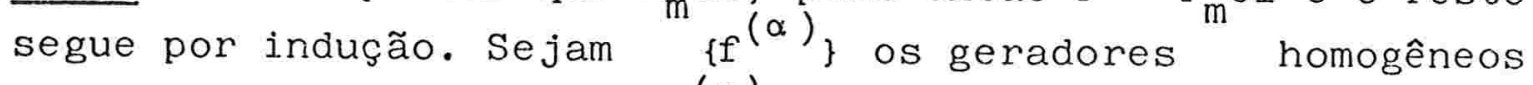
de I e seja d $\alpha$ ograu de $f^{(\alpha)}$.
Pondo $F=\left[A^{(\alpha)} f^{(\alpha)}\right.$ e decompondo cada $A^{(\alpha)}$, $A^{(\alpha)}=\sum_{j=0}^{b} A_{j}(\alpha)$, onde $A_{j}(\alpha)$ é homogêneo de grau $j$ temos: $F_{m}=\sum A_{m-d_{\alpha}}^{j=0} f^{(\alpha)}$, donde $F_{m} \in I$. C.Q.D.

Um elemento $\bar{g}$ de $k_{h}[V]$ é dito homogêneo de grau d se existir um polinômio homogêneo $g$ de grau d : em $k\left[x_{0}, x_{1}, \ldots, x_{n}\right]$ tal que $\bar{g}=g+I_{k}(V)$. 
Se $\overline{\mathrm{f}}$ e $\overline{\mathrm{g}}$ são elementos de $\mathrm{k}_{\mathrm{h}}[\mathrm{V}$ ]homogêneos e de mes mo grau d, então $\bar{f} / \bar{g}$ efetivamente define uma função em $V$ (se $g \neq 0)$ pois

$$
\frac{f(\lambda x)}{g(\lambda x)}=\frac{\lambda^{d} f(x)}{\lambda^{d} g(x)}=\frac{f(x)}{g(x)}
$$

de modo que $f(x) / g(x)$ independe da escolha de coordenadas homogêneas, e claramente independe da escolha dos represen tantes $f$ e g das classes $\bar{f}$ e $\bar{g}$.

o corpo de funções de $V$, denotado $k(V)$, é defini do como o subconjunto de $k_{h}(V)$ que consiste das funções $\phi$ para as quais existem $f, g \in k_{h}[V]$ elementos homogêneos de mesmo grau, tais que $\phi=\bar{f} / \bar{g}$. Os elementos de $k(V)$ são cha mados de funções racionais em $V$.

Seja PeV, zek(V). Dizemos que z está definida em $P$ se $z=\bar{f} / \bar{g}$ para certos $\bar{f}, \bar{g}$ homogêneos de mesmo grau e $g(P) \neq 0$. Seja $O_{p}(V)$ o subconjunto de $k(V)$ que consiste das funções que estão definidas em $P$. Então $O_{p}(V)$ é um sub anel de $k(V)$ e é um anel local cujo único ideal maximal é $M_{p}=\{z: z=\bar{f} / \bar{g}, g(P) \neq 0, f(P)=0\}$.

Seja $\mathrm{V}$ una $\mathrm{K} / \mathrm{k}$-variedade afim, e I o seu ideal em $\mathrm{k}\left[\mathrm{X}_{1}, \ldots, \mathrm{X}_{\mathrm{n}}\right]$ (o caso projeto é inteiramente análogo). Seja $\bar{I}=I \otimes K \subset K\left[X_{1}, \ldots, X_{n}\right]$ o ideal gerado por $I$. De finimos $\bar{V}=V_{K}(\bar{I})$ como a variedade correspondente a $V$ obtida por extensão de escalares. Conjuntisticamente, $V=\bar{V}$.

No que se segue vamos explorar um pouco as rela ções entre variedades afins e variedades projetivas. Por sim plicidade vamos supor que $k=K$ é um corpo algebricamente fé chado. Os n-espaços afim e projetivo sobre $K$ serão denotados respectivamente $\mathbb{A}^{\mathrm{n}}$ a $\mathbb{P}^{\mathrm{n}}$. 
Se $F$ é um polinômio homogêneo de $\mathrm{K}\left[\mathrm{X}_{0}, \mathrm{X}_{1}\right.$, $\left.\ldots . x_{n}\right], \quad$ definimos $F_{*} \operatorname{eK}\left[x_{1}, \ldots, x_{n}\right]$ pondo $F_{*}=F\left(1, X_{1}, X_{2}, \ldots, x_{n}\right)$. Reciprocamente para qualquer po linômio $f \in K\left[X_{1}, X_{2}, \ldots, X_{n}\right]$ de grau $d$, pomos $f=f_{0}+$ $f_{1}+\ldots+f_{d}$ onde $f_{i}$ é um polinômio homogêneo de grau i, e definimos $f^{*} \in K\left[x_{0}, x_{1}, \ldots, x_{n}\right]$ como

$$
f^{*}=x_{0}^{d} f_{0}+x_{0}^{d-1} f_{1}+\ldots+f_{d}=x_{0}^{d} f\left(x_{1} / x_{0}, \ldots, x_{n} / x_{0}\right)
$$

e $f^{*}$ é um polinômio homogêneo de grau d.

Esses processos podem ser entendidos como uma des homogenização e homogenização de polinômios em relação à vâ riável $x_{0}$. E imediata a

PROPOSIÇAO 2. Se F, G são polinômios homogêneos de $K\left[X_{0}, X_{1}, \ldots, X_{n}\right]$ e $f, g$ polinômios arbitrários de $\mathrm{K}\left[\mathrm{X}_{1}, \ldots, \mathrm{X}_{\mathrm{n}}\right]$ então:

(a) $(F G)_{*}=F_{*} G_{*} ;(f g)^{*}=f^{*} g^{*}$

(b) Se $r$ é a maior potência de $x_{0}$ que divide $F$, então $x_{0}^{r}\left(F_{*}\right)^{*}=F$ e $\left(f^{*}\right)_{*}=f$

(c) $(F+G)_{*}=F_{*}+G_{*} ; x_{0}^{t}(f+g)^{*}=x_{0}^{r_{f}^{*}}+x_{0}^{s} g^{*}$ onde $t=r+s-\operatorname{grau}(f+g)$,

$r=\operatorname{grau}(g)$,

$\mathbf{s}=\operatorname{grau}(f)$.

Definimos anteriormente os subconjuntos $U_{i}$ de $\mathbf{P}^{\mathrm{n}}$ como $U_{i}=\left\{\left(x_{0}, x_{1}, \ldots, x_{n}\right) \in p^{n}: x_{i} \neq 0\right\}$. E claro que ca da $\mathrm{PeU}_{i}$ possui um único conjunto de coordenadas homogêneas da forma: $P=\left(x_{0}, \ldots, x_{i-1}, x_{i+1}, \ldots, x_{n}\right)$. As coordena 
das $\left(x_{1}, \ldots, x_{i-1}, x_{i+1}, \ldots, x_{n}\right)$ são chamadas

coordena das' não-homogêneas para $P$ em relação à $U_{i}$ •

Definindo $\psi_{i}: \mathbb{A}^{n}+U_{i}$ por $\psi_{i}\left(x_{1}, x_{2}, \ldots, x_{n}\right)=\left(x_{1}, x_{2}, \ldots, x_{i-1}\right.$, $\left.1, x_{i+1}, \ldots, x_{n}\right), \psi_{i}=\phi_{i}^{-1}$, onde $\phi_{i}$ é a aplicação $j a ́$ definida no início do $\S 2$, podemos considerar $\mathbb{A}^{\mathrm{n}}$ como sub conjunto de $\mathbb{P}^{n}$. Pomos $\mathrm{H}_{\infty}=\mathbb{P}^{\mathrm{n}}-\mathrm{U}_{0}=\left\{\left(\mathrm{x}_{0}, \ldots, \mathrm{X}_{\mathrm{n}}\right): \mathrm{X}_{0}=0\right.$ \} e é usual dizer que $H_{\infty}$ é o hiperplano no infinito.

A correspondência $\left(0, x_{1}, \ldots, x_{n}\right) \leftrightarrow\left(x_{1}, \ldots, x_{n}\right)$ mostra que $H_{\infty}$ pode ser identificado com $\mathbf{P}^{n-1}$.

Seja $V$ uma variedade afim em $\mathbb{A}^{n}, \quad I=I_{K}(V) \circ$ seu ideal em $K\left[X_{1}, X_{2}, \ldots, X_{n}\right]$. Seja $I^{*} \circ$ ideal em $K\left[X_{0}, x_{1}, \ldots, x_{n}\right]$ gerado pelos $f^{*}$, fGI. Então $I^{*}$ é um ideal homogêneo, e definimos $V^{*} \operatorname{com~} V_{K}\left(I^{*}\right)$. Reciprocamente, seja $V$ uma variedade projetiva em $\mathbb{P}^{n}, I=I_{K}(V)$ o seu ideal homogêneo. Analogamente seja $I_{*} \quad o$ ideal em $K\left[X_{1}, X_{2}, \ldots, X_{n}\right]$ gerado pelos $f_{*}$, feI. Definimos $V_{*} \underline{\text { o }}$ mo $V_{K}\left(I_{*}\right) \subset \mathbb{A}^{n}$.

PROPOSIÇÃO 3. Nas condições acima valem:

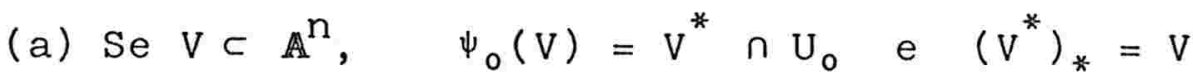

(b) Se $V \subset W \subset \mathbb{A}^{n}$, então $V^{*} \subset W^{*} \subset \mathbb{P}^{n}$

Se $V \subset W \subset \mathbf{P}^{n}$, então $V_{*} \subset W_{*} \subset \mathbb{A}^{n}$

(c) Se $V$ é irredutivel em $\mathbb{A}^{n}$, então $V^{*}$ será irre dutivel em $\mathbf{P}^{\mathrm{n}}$.

(d) Se V $=\underset{i}{U} V_{i}$ é a decomposição de $V$ em suas com ponentes irredutiveis, em $\mathbb{A}^{n}$, então $v^{*}=U_{i} V_{i}{ }^{*}$ é a decomposição de $V^{*}$ em suas componentes irredutíveis, em $\mathbf{P}^{n}$. 
(e) Se $V \subset \mathbb{A}^{n}$, então $V^{*}$ é o menor fechado de $\mathbb{P}^{n}$ que contém $\psi_{0}(V)$.

PROVA. Se $\left(x_{1}, x_{2}, \ldots, x_{n}\right) \in V$, sua imagem por $\psi_{0}$ será $\left(1, x_{1}, \ldots, x_{n}\right)$ eU $U_{0}$. Vamos mostrar que $\left(1, x_{1}, \ldots, x_{n}\right)$ está em $V^{*}=V_{K}\left(I^{*}\right)$. Para tanto é suficiente verificar que se feI, então $f^{*}\left(1, x_{1}, \ldots, x_{n}\right)=0$, o que é claro. A recí proca é análoga, o que prova que $\psi_{0}(V)=V^{*} \cap U_{0} \cdot 0$ item (b) da proposição 2 prova que $\left(V^{*}\right)_{*}=V$. Isso prova (a). A parte (b) é óbvia.

Para provar (e), suponhamos que W seja um fechado de $\mathbb{P}^{\mathrm{n}}$ que contém $\psi_{0}(V)$. Se $F \in I(W)$ então $F_{*} \in I(V), \quad$ donde $\mathrm{F}=\mathrm{X}_{0}^{\mathrm{r}}\left(\mathrm{F}_{*}\right)^{*} \in I(\mathrm{~V})^{*}$ donde $\mathrm{I}(\mathrm{W}) \subset \mathrm{I}(\mathrm{V})^{*}$, e portanto $\mathrm{W} \supset \mathrm{V}^{*}$, o que prova (e). Para provar (c), note-se que se $V \subset \mathbb{A}^{n}$, $I=I(V)$, então um polinômio homogêneo $F$ pertencerá a $I^{*}$ se e só se $F_{*} \in I$. Se I for primo, então é claro que $I^{*}$ será primo, o que prova (c).

Para provar (d), por (e), se $v_{i}$ é irredutivel, $v_{*}{ }^{*}$ será irredutivel e por (a), (b), se $v_{i} \not^{i} v_{j}$ então $v_{i}^{*} \not \subset v_{j}^{*}$, e isso prova (d).

Se $V$ é um fechado de $\mathbb{A}^{\mathrm{n}}, \mathrm{V}^{*} \subset \mathbb{P}^{\mathrm{n}}$ é chamado o fecho projetivo de V. Se $f \in K_{h}\left[V^{*}\right]$ é um elemento homogêneo de grau d, podemos definir $f_{*} e K[V]$ assim: tomamos um polinômio homogê neo de grau $d, F \in K\left[X_{0}, X_{1}, \ldots, X_{n}\right]$ tal que $f=F+I_{K}\left(V^{*}\right)$ e tomamos $f_{*}=F_{*}+I_{K}(V)$. E claro que a definição independe da particular escolha de F.

Definimos assim um isomarfismo natural $\alpha: K\left(V^{*}\right)+K(V)$ dado por $\alpha(f / g)=f_{*} / g_{*}$. $\alpha$ está bem definido e é um marfís mo de corpos e $\alpha$ é sobrejetor pois se $1 / \mathrm{heK}(\mathrm{V}), \operatorname{grau}(1)=\mathrm{m}$, $\operatorname{grau}(\mathrm{h})=\mathrm{n}$, então $1^{*}, \mathrm{~h}^{*} \mathrm{GK}\left[\mathrm{V}^{*}\right]$ e $\frac{\mathrm{x}_{0}^{\mathrm{n}-\mathrm{m}} 1^{*}}{\mathrm{~h}^{*}} \mathrm{G} \mathrm{K}\left(\mathrm{V}^{*}\right)$ e sua ima gem é precisamente $1 / \mathrm{h}$. 
Se PeV, então $\alpha$ induz um isomorfismo

entre

$\mathrm{O}_{\mathrm{P}}\left(\mathrm{V}^{*}\right)$ e $\mathrm{O}_{\mathrm{P}}(\mathrm{V})$ (estamos aqui pensando em $\mathrm{R}$ como um ponto de $\mathrm{V} *$ via $\left.\psi_{0}\right)$.

Até agora definimos variedades afins e projetivas e examinamos um pouco as relações entre elas. Porém ainda não definimos transformações entre duas variedades quaisquer. No que se segue continuaremos no mesmo contexto que antes, isto $\dot{e}, k=K$ é um corpo algebricamente fechado.

DEFINIÇÃO. Seja $V$ uma variedade afim de $\mathbb{A}^{n}$. Uma função $f: V+K$ é regular no ponto $\mathrm{PEV}$ se existir uma vizinhança aberta $U$ com $P \in U \subseteq V$ e polinômios $g, h \in K\left[X_{1}, \ldots, X_{n}\right]$ tal que $h$ não se anula em nenhum ponto de $U$ e $f=g / h$ em $U$. Di zemos que $f$ é regular em $V$ se for regular em todo ponto de V.

DEFINIĈ̃̃O: Seja $V$ uma variedade projetiva em $\mathbb{P}^{\mathrm{n}}$. Uma função $f: V+K$ é regular no ponto $P \in V$ se existir uma vizinhança aberta $U$ com $P \in U \subseteq V$, e polinômios homogêneos de mesmo grau, $g, h \in K\left[x_{0}, x_{1}, \ldots, x_{n}\right]$ tais que $h$ não se anula em $U$ e $f=g / h$ em $U$. Dizemos que $f$ é regular em $V$ se for regu lar em todos os pontos de V.

Como ficou claro até aqui, somente temos interes se em variedades afins ou projetivas que sejam irredutiveis. De modo que, para tudo o que seguirá neste e nos demais capj. tulos, estamos supondo que, salvo menção contrária, toda variedade afim ou projetiva é irredutível.

DEFINIÇÃO: Sejam X e Y duas variedades sobre K. (Podem ser ambas afins, projetivas ou uma afim e outra projetiva). Um morfismo $\phi: X+Y$ é uma aplicação contínua tal que para todo aberto $V \subseteq Y$ e para toda função regular $f: V+K$, a função $f \circ \phi \mid \phi^{-1}(V)+K$ é regular. 
Temos em particular a noção de isomorfismo de varie dades $\phi: X+Y$, como um morfismo que admite um mofirsmo inverso $\psi: Y+X$ com $\psi \circ \phi=i d_{X} e \phi \circ \psi=i d_{Y}$.

Em vista da definição acima, é fácil ver que $\mathbb{A}^{\mathrm{n}}$ e $U_{o}$ são isomorfos como variedades.

\section{$\$ 3$ - Curvas não Singullares}

Já vimos que se $\mathrm{V}$ é uma variedade, $\mathrm{k}(\mathrm{V}) / \mathrm{k}$ é uma extensão finitamente gerada e a dimensão de $\mathrm{V}$ é igual 0.0 grau de transcendência de $k(V) / k$. Seja $K$ um corpo algebrica mente fechado, $\mathrm{K} \supset \mathrm{k}$. Uma $\mathrm{K} / \mathrm{k}$-variedade (afim ou projetiva) de dimensão 1 foi chamada curva (afim ou projetiva). Se V é uma curva sobre $\mathrm{k}$, o seu corpo de funções $\mathbb{K}=\mathrm{k}(\mathrm{V})$ é um corpo de funções algébricas de uma variável sobre $\mathrm{k}$ pois, co mo $\mathrm{V}$ é curva, a extensão $\mathrm{k}(\mathrm{V}) / \mathrm{k}$ tem grau de transcendência 1 isto é, existe um elemento $x e k(V)$ transcendente sobre $k$ tal que $k(V) / k(x)$ é algébrica. Como $k(V) / k$ é finitamente ge rada, $k(V) / k(x)$ será portanto finita.

No que se segue, suporemos que $\mathrm{k}=\mathrm{K}$ seja um corpo algebricamente fechado, e em lugar de $\mathbb{A}_{k}^{n}$ e $\mathbb{P}_{k}^{n}$ escreveremos somente $\mathbb{A}^{\mathrm{n}}$ e $\mathbb{P}^{\mathrm{n}}$.

PROPOSIÇÃO 1. Seja $V \cdot \subset \mathbb{A}^{\mathrm{n}}$ uma variedade afim sobre $\mathrm{k}$ e $\quad \mathrm{V}^{*}$ o seu fecho projetivo em $\mathbb{P}^{n}$.

(a) $\operatorname{dim} V^{*}=\operatorname{dim} V$

(b) $\operatorname{dim} V=0$ se e só se $V$ for um ponto.

(c) toda subvariedade própria de uma cruva afim é um ponto. 
PROVA. (a) decorre do fato de que $\mathrm{V}$ e $\mathrm{V}^{*}$ possuem corpos de funções isomorfos. Para provar (b), suponhamos que dim $V=0$. Então k(V)/k é uma extensão algébrica, e como k é algebricamente fechado, $\mathrm{k}(\mathrm{V})=\mathrm{k}$, donde $\mathrm{k}[\mathrm{V}]=\mathrm{k}$, o que acarreta $I_{k}(V)$ maximal, e assim $V=\{P\}$. A recíproca é clara.

Para provar (c), seja $W$ uma subvariedade própria de $V$, e seja $R=k[V], P$ o ideal primo de $R$ correspondente a W. (isto é $P$ é o kernel do epimorfismó natural. $n: k[V]+k[W])$

(c) estará provado se mostrarmos que $R / P \simeq k$.

Consideremos a aplicação natural de $k$ em R/P. Pro varemos que ela é sobrejetora.

Suponhamos que existe $x \in R$ tal que $x+P$ não seja imagem de nenhum elemento de $\mathrm{k}$.

Como $W$ é própria, $\mathrm{P} \neq 0$, e portanto existe $y \in P, y \neq 0$. Como $\circ$ grau de transcendência de $k(V)$ sobre $k$ é 1, y satisfaz um polinômio irredutível com coeficientes em $k(x), F(x, y)=\sum_{i} a_{i}(x) y^{i}=0$. Então decorre que $a_{o}(x) \neq 0$ e portanto $a_{0}(x) \in P$. Donde $x+P G R / P$ satisfaz o polinômio $a_{0} \in \mathrm{k}[\mathrm{x}]$.

Mas como $\mathrm{k}$ é algebricamente fechado, $\mathrm{x}+\mathrm{P} \in \mathrm{k}$, ou seja, a aplicação dada é sobrejetora. C.Q.D.

Estamos nos preparando para provar o resultado mais importante deste capitulo, o teorema 2 .

Vamos agora relembrar o contexto em que estamos: $\mathrm{k}$ é um corpo algebricamente fechado, e se $\mathrm{V}$ é uma variedade afim ou projetiva sobre k, V é sempre irredutivel. 
DEFINIÇÃo. Seja C uma cruva arbitrária e $P \in$ C. Dizemos que $P$ é não singular se $O_{P}(\mathbb{C})$ for um anel de valorização discre ta. A curva c é dita não singular se todos os seus pontos fó rem não singulares.

Em geral, se $Y \subseteq \mathbb{A}^{n}$ é uma variedade afim e $f_{1}, \ldots, f_{m} \in k\left[x_{1}, x_{2}, \ldots, x_{n}\right]$ é um conjunto de geradores de $I_{K}(Y)$, diz-se que $Y$ é não singular no ponto $P \in Y$ se o posto da matriz $\|\left(\partial f_{i} / \partial X_{j}\right)(P)||$ é $n-r$ onde $r=\operatorname{dim} Y$. Y é dita não singular se for não singular em todos os seus pontos.

Essa definição é boa (pode-se mostrar que ela independe do particular conjunto de geradores escolhido), porém tem a desvantagem de depender (aparentemente) da imersão de Y num espaço afim. Entretanto, prova-se ([5], pg.32) que $Y$ é não singular em $P \in Y$ se e só se o anel local $O_{P}(Y)$ é um anel local regular, ou seja, se $\operatorname{dim} O_{P}(Y)=\operatorname{dim}{ }_{k} M_{P} / M_{P}^{2}$. Em particular, se $Y$ for uma curva afim, prova-se que $O_{P}(Y)$ é um anel local regular se e só se for um anel de valoriza ção discreta ([2], pg.94). Sendo então um conceito intrín seco, extendemos a definição de não-singularidade para variedades projetivas, via a regularidade do anel local $\mathrm{O}_{\mathrm{P}}(\mathrm{Y})$. Como estamos interessados em curvas, a definição da da acima é a mais adequada aos nossos propósitos.

OBSERVAÇÃo. A aplicação $\left(x_{1}, \ldots, x_{n}\right) \longmapsto\left(0, x, \ldots, x_{n}\right)$ dá um isomorfismo de variedades entre $\mathbb{P}^{n-1}$ e $\mathrm{H}_{0}=\mathbb{P}^{\mathrm{n}}-\mathrm{U}_{0} \subset \mathbb{P}^{\mathrm{n}}$. Se uma variedade $\mathrm{V}$ em $\mathbb{P}^{\mathrm{n}}$ estiver contida em $\mathrm{H}_{0}, \mathrm{~V}$ é isoforma a uma variedade de $\mathbf{P}^{\mathrm{n}-1}$.

Assim, dada uma variedade projetiva, existe um $n$ de modo que ela é isomorfa a uma subvariedade $V$ de $\mathbb{P}^{n}$ que não está contida em nenhum hiperplano.

TEOREMA 1. Seja C uma curva projetiva definida sobre k. Se 
ja L um corpo arbitrário contendo $\mathrm{k}(\mathbb{C})$ e $\mathrm{A}$ um anel de valori zação discreta de $\mathrm{L}$ que contém $\mathrm{k}(\mathrm{A} \supset \mathrm{k})$, com ideal maximal M. Suponhamos que $A \not \vec{k}(\mathbf{C})$. Então existe um ponto PeC tal que $A \supset O_{P}\left(\mathbb{C}^{\prime}\right)$ e $M \supset M_{P}$, onde $M_{P} \dot{e} \circ$ ideal maximal de $\mathrm{O}_{\mathrm{P}}$

PROVA. Em vista da observação anterior podemos supor que $\mathbb{C}^{*}$ seja uma variedade em $\mathbb{P}^{n}$ com $\mathbf{C} \cap U_{i} \neq 0, i=0,1, \ldots, n$. Então em $\mathrm{k}_{\mathrm{h}}[\mathbf{C}]=\mathrm{k}\left[\mathrm{x}_{0}, \mathrm{x}_{1}, \ldots, \mathrm{x}_{\mathrm{n}}\right] / \mathrm{I}_{\mathrm{k}}(\mathbb{C})=\mathrm{k}\left[\mathrm{x}_{0}, \mathrm{x}_{1}, \ldots, \mathrm{x}_{\mathrm{n}}\right]$ $x_{i}=x_{i}+I_{k}(\mathbb{C})$, cada $x_{i} \neq 0$. Observe que $x_{i} / x_{j} \in k(\mathbb{C})$. Seja $N=\max _{i, j}\left\{v\left(x_{i} / x_{j}\right)\right\}$, onde $v$ é a valorjzação associada do anel A. Fazendo uma mudança de coordenadas se necessário, podemos supor que $N=v\left(x_{j} / x_{0}\right)$ para certo $j$. Então para todo $i, v\left(x_{i} / x_{0}\right)=v\left(\left(x_{j} / x_{0}\right) \cdot\left(x_{i} / x_{j}\right)\right)=N-v\left(x_{j} / x_{i}\right) \geq 0$.

Seja $\mathbb{C}_{*}$ a curva afim correspondente à $\mathbb{C}^{\prime} \cap U_{0}$.

Seja $\theta: k\left[x_{1}, \ldots, x_{n}\right]+k\left[x_{1} / x_{0}, \ldots, x_{n} / x_{0}\right] \quad a$ aplicação natural que leva $x_{i}+x_{i} / x_{0}$. E claro que $\theta$ é um epimorfismo de anéis. Se feKer $\theta, f\left(x_{1} / x_{0}, \ldots, x_{n} / x_{0}\right) e I_{k}(\mathbb{C})$, donde, se $r=\operatorname{grau}(f), x_{0}^{r} f\left(x_{1} / x_{0}, \ldots, x_{n} / x_{0}\right) e I_{k}\left(\mathbb{C}^{*}\right)$, donde $f^{*} \in I_{k}(c)$, donde $\left(f^{*}\right)_{*} \in I_{k}(\mathbb{C})_{*} \subset I_{k}\left(\mathbb{C}_{*}\right)$. Mas pela proposição 2, (b), segue que $f \in I_{k}\left(\mathbf{c}_{*}\right)$. Ou seja, Ker $\theta \subset I_{k}\left(\mathbf{c}_{*}\right)$. Mas claramente $I_{k}\left(\mathbb{C}_{*}\right) \subset \operatorname{Ker} \theta$, donde provamos que

$$
\mathrm{k}\left[\mathbb{C}_{*}\right] \simeq \mathrm{k}\left[\mathrm{x}_{1} / \mathrm{x}_{0}, \ldots, \mathrm{x}_{\mathrm{n}} / \mathrm{x}_{0}\right]
$$

Como $v\left(x_{i} / x_{0}\right) \geq 0$, e $A \supset k$, segue das considera ções acima que $A \supset k\left[\mathbf{C}_{*}\right]$. Seja $J=M \cap k\left[\mathbf{C}_{*}\right]$. Então $J$ é um ideal primo e portanto corresponde a uma subvariedade $\mathrm{W} \subseteq \mathbf{C}_{*}$.

Se $W=\mathbf{C}_{*}$, então $\mathrm{J}=(0)$ e portanto todo elemen to não nulo de $\mathrm{k}\left[\mathbf{C}_{*}\right.$ ]é inversível em A. Nesse caso teríamós $k\left(\mathbf{C}_{*}\right) \subset A$, ou seja, $k(\mathbf{C}) \subset A$, contra a hipótese. Logo, pela 
proposição $1,(\mathrm{~b}), \S 3, W=\{\mathrm{P}\}$ e se $\mathrm{f} / \mathrm{geO}_{\mathrm{P}}\left(\mathbf{C}_{*}\right)=\mathrm{O}_{\mathrm{P}}\left(\mathbb{C}^{u}\right)$, $f, g e k\left[C_{*}\right], g \notin J$, assim $v(f / g)=v(f)-v(g)$, mas como $g \notin M, v(g)=0$, donde $v(f / g) \geq O$, donde $A \supset O_{P}\left(\mathbf{C}_{*}\right)=O_{P}(C)$ e claramente $\mathrm{M} \supset \mathrm{M}_{\mathrm{P}}$. C.Q.D.

COROLARIO 1. Seja $\mathbb{C}$ uma curva projetiva não singular defí nida sobre k. Então existe uma correspondência bijetora en tre os pontos de $\mathbb{C}$ e os anéis de valorização discreta de $\mathrm{k}$ (C) que contém $\mathrm{k}$. Se PeC, o correspondente anel de valoriza ção discreta é $\mathrm{O}_{\mathrm{P}}(\mathbb{C})$.

PROVA. Como a curva $\mathbb{C}$ é não singular, $\mathrm{O}_{\mathrm{P}}(\mathbb{C})$ é um anel de valorização discreta. Suponhamos que $O_{P}(\mathbb{C})=O_{Q}(\mathbb{C})$ para cer tos pontos $P, Q G C$. Como sempre existe um hiperplano $H \subset \mathbb{P}^{\bar{n}}$ tal que $P, Q \notin H$, posso considerar que $C$ é afim, e nesse caso $\mathrm{O}_{\mathrm{P}}(\mathbf{C})=\{\mathrm{f} / \mathrm{g}: \mathrm{f}, \mathrm{g} \in \mathrm{k}[\mathbf{C}] ; \mathrm{g}(\mathrm{P}) \neq \mathrm{O}\}=\mathrm{k}[\mathbf{C}]_{\mathrm{M}}$, onde $\mathrm{k}[\mathbf{C}]_{\mathrm{M}}$ in dica o localizado de $\mathrm{k}[\mathbf{C}]$ em relação ao ideal maximal $M=\{f e k[\mathbb{C}]: f(P)=O\}$. Analogamente $O_{Q}(\mathbb{C})=k[C]_{N}$, onde $N$ é o ideal maximal $N=\{\operatorname{gek}[\mathbb{C}]: g(Q)=0\}$. Assim, se

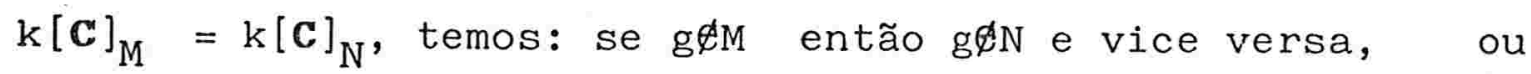
seja, $M=N$. Os ideais $M$ e $N$ de $k[C]$ correspondem a ideais $\operatorname{maximais} \bar{M}$ e $\bar{N}$ de $k\left[X_{1}, X_{2}, \ldots, X_{n}\right], \quad \bar{N}=I_{k}(\{Q\}) \quad$ e $\bar{M}=\left\{f \in k\left[X_{1}, X_{2}, \ldots, X_{n}\right]: f(P)=0\right\}=I_{k}(\{P\})$. Mas en tão $\bar{M}=\bar{N}$, e, em geral vale: se $V$ e $W$ são fechados de $\mathbb{A}_{k}^{n}$, então $V=W$ se e só se $I_{k}(V)=I_{k}(W)$. (lembrando que $k$ é al grbricamente fechado). Donde segue, por esse resultado que $P=Q$. Agora, se $A$ é um anel de valorização discreta de $k(C)$ que contém $k$, pelo teorema 1 existe Pec tal que $A \supset \mathrm{O}_{\mathrm{P}}(\mathbf{C})$. Mas pela maximalidade dos anéis de valorização discreta (le ma $3, \S 2$, Cap. I) segue que $A=O_{P}(\mathbf{C})$. C.Q.D.

Tendo provado esse corolário, vamos agora conside rar o caso em que C é uma curva projetiva definida sobre um 
corpo finito $\mathbb{F}_{q}$. Diremos $\mathbf{C} / \mathbb{F}_{q}$ é não singular se a curva obti da pela extensão de escalares à $\overline{\mathbb{F}}_{q} \circ$ for.

Já vimos que $\overline{\mathbb{F}}_{\mathrm{q}} / \mathbb{F}_{\mathrm{q}}$ é uma extensão de Galois. Seja $G=\operatorname{Gal}\left(\overline{\mathbb{F}}_{\mathrm{q}} / \mathbb{F}_{\mathrm{q}}\right)$. Se $\mathrm{J}$ é um ideal homogêneo de $\mathbb{F}_{q}\left[X_{0}, X_{1}, \ldots, X_{n}\right]$, consideremos a variedade $Y=V_{\bar{F}_{q}}(J) \subset$ $\mathbb{P}_{\bar{P}_{\mathrm{q}}}^{n}$. Então o grupo de Galois $G$ age natüralmerite em $\mathrm{Y}$, e cada uma das órbitas é finita, pelo comentário que se segue à proposição 6 do $\S 1$ do Capítulo I. Uma órbita da ação de G em Y é chamada de ponto fechado de $Y$.

TEOREMA 2. Seja C uma curva projetiva não singular definida sobre um corpo finito $\mathbb{F}_{\mathrm{q}}$. (Isto é, $\mathbf{C}$ é uma $\overline{\mathrm{F}}_{\mathrm{q}} / \mathrm{F}_{\mathrm{q}}$ - variedade projetiva de dimensão 1). Então existe uma correspondência. bijetora entre os pontos fechados de $\mathbf{C}$ e os anéis de valori zação discreta de $\mathbb{F}_{q}\left(\mathbb{C}^{\prime}\right)$ que contém $\mathbb{E}_{q}$, que a cada ponto fé chado $p$ de $\mathbf{C}$ associa o anel $\mathrm{O}_{\mathrm{p}}(\mathrm{C})$, onde $\mathrm{Pep}$.

PROVA. Seja I o ideal homogêneo de $\mathbb{C}$ e $\bar{I}=I \otimes \bar{F}_{q} \subset$ $\subset \overline{\mathbb{F}}_{\mathrm{q}}\left[\mathrm{x}_{0}, \mathrm{x}_{1}, \ldots, \mathrm{x}_{\mathrm{n}}\right]$ a extensão de $\mathrm{I}$ (vide pag 28). Pon do $\overline{\mathbf{C}}=\mathrm{V}_{\overline{\mathbf{F}}_{\mathrm{q}}}(\overline{\mathrm{I}})$, temos que, como conjuntos, $\mathrm{C}=\overline{\mathrm{C}}$. Se

PEC $=\overline{\mathbf{C}}$, por definição $O_{P}(\bar{C})$ é um anel de valorização dis creta de $\overline{\mathbb{F}}_{\mathrm{q}}(\overline{\mathbf{C}})$. Seja $\bar{V}=\bar{V}\left(\overline{\mathbb{F}}_{\mathrm{q}}(\overline{\mathrm{C}}) / \overline{\mathbb{F}}_{\mathrm{q}}\right)$ o conjunto dos anéis de valorização discreta de $\overline{\mathbf{F}}_{\mathrm{q}}^{\mathrm{q}}(\overline{\mathbf{C}})$ que contém $\mathrm{q}_{\mathrm{q}}$ e $\mathrm{V}=\mathrm{V}\left(\mathrm{F}_{\mathrm{q}}(\mathbf{C}) / \mathbb{F}_{\mathrm{q}}\right)$ o conjunto dos anéis de valorização discre ta de $\mathbf{F}_{\mathrm{q}}(\mathbf{C})$ que contém $\mathbb{F}_{\mathrm{q}}$. Consideremos a ação de $\mathrm{G}=\mathrm{Gal}\left(\overline{\mathbb{F}}_{\mathrm{q}} / \mathbb{F}_{\mathrm{q}}\right)$ em $\bar{V}$ definida assim: se $\sigma G G, O_{\mathrm{P}}(\overline{\mathrm{C}}) \mathrm{e} \overline{\mathrm{V}}$, $\sigma \cdot O_{P}(\bar{C})=O_{\sigma(P)}(\bar{C})$. 
Seja $R: \bar{V}+V$ a aplicação natural dada por $R\left(O_{P}(\bar{C})\right)=O_{P}(\bar{C}) \cap \mathbb{F}_{q}(C)$. Se pensarmos em $\bar{V}$ como $\circ$ conjunto das valorizações discretas de $\overline{\mathbb{F}}_{Q}(\bar{C})$ que se anulam sobre $\bar{F}_{q}$, R é simplesmente a restrição usual de funções, pois como $\overline{\mathbf{F}}_{\mathrm{q}} / \mathrm{F}_{\mathrm{q}}$ é separável, pelo lema 5 da pag 16 , não há rami ficação.

Pelo teorema 1 do $\S 2$ do Cap. I, $R$ é uma aplicação sobrejetora. Além disso, se oeg então $\mathrm{O}_{\mathrm{P}}(\overline{\mathrm{C}}) \cap \mathbb{F}_{\mathrm{q}}(\mathbf{C})=$ $o_{\sigma(P)}(\widetilde{C}) \cap F_{q}(C)$ pois se $f e \mathbb{F}_{q}(\mathbb{C})$ está definida em $P$, f estará definida em $\sigma(P)$ e reciprocamente. Assim, se GlV denota $O$ conjunto das órbitas da ação de $G$ em $\bar{V}$, a aplicação $R$ induz uma aplicação $\hat{R}: G \backslash \bar{V} \rightarrow V$ que é sobrejetora. Mas $\hat{R}$ é também injetora pois, pelo teorema 2 do $\$ 2$ do Cap. I, se veV e $v_{1}$ e $v_{2}$ são elementos de $\bar{V}$ que extendem $v$ então existe o eG tal que $v_{1}=v_{2} \circ \sigma$. Ou seja, $\hat{R}$ é uma bijeção. O corolário 1 deste $\S$ nos garante que há uma correspondência bijetora $\Phi$ entre os pontos de $\overline{\mathbb{C}}=\mathrm{C}$ e os elementos de $\bar{V}$, dada por $\Phi(P)=O_{P}(\bar{C})$.

Já vimos que $G$ age naturalmente em $\mathbb{C}=\overline{\mathbf{C}}$ e é fá cil ver que $\Phi$ comuta com essa ação, isto é

$$
\Phi(\sigma(P))=O_{\sigma(P)}(\bar{C})=\sigma \cdot O_{P}(\bar{C})=\sigma \cdot \Phi(P)
$$

e isso implica que $\Phi$ leva uma órbita da ação de $\mathrm{G}$ em $\mathrm{C}=\overline{\mathrm{C}}$ numa órbita da ação de $G$ em $\bar{V}$. Assim, se G\C denota o con junto dos pontos fechados de C, $\Phi$ induz uma bijeção $\hat{\Phi}: G \backslash C+$ $G \backslash \bar{V}$, donde a composição:

$$
G \backslash C \stackrel{\hat{\Phi}}{\rightarrow} \quad G \backslash \bar{V} \stackrel{\hat{R}}{\rightarrow} V
$$

é uma aplicação bijetora.

C.Q.D. 
Se v é uma valorização discreta de um corpo $\mathrm{K}, \mathrm{K} \supset \mathrm{k}$, trivial em $\mathrm{k}$ e $\mathrm{A}_{\mathrm{V}}$ e $\mathrm{M}_{\mathrm{v}}$ são respectivamente o seu anel de valorização e o seu ideal maximal, o corpo $\mathrm{A}_{\mathrm{v}} / \mathrm{M}_{\mathrm{V}}$ é dito corpo de restos de v (vide $\S 2$, Cap. I) e a sua dimen são como k-espaço vetorial é denotada d(v).

Em particular, no nosso caso, se p é um ponto fe chado de $\mathbf{C}$ e $|p|$ designa o número de elementos da órbita $p$, vamos mostrar que a correspondência que leva $p$ na valoriza ção $v_{p}$ de $F_{q}(\mathbb{C})$, trivial sobre $\mathbb{F}_{q}$, preserva o grau, isto é, $|p|=\alpha\left(v_{p}\right)$.

Seja então $p$ um ponto fechado de $\mathbb{C}$ de tamnaho $r$; $|p|=r, p=\left\{P, P^{q}, \ldots, P^{q^{r-1}}\right\}$ onde $P=\left(x_{0}, x_{1}, \ldots, x_{n}\right)$

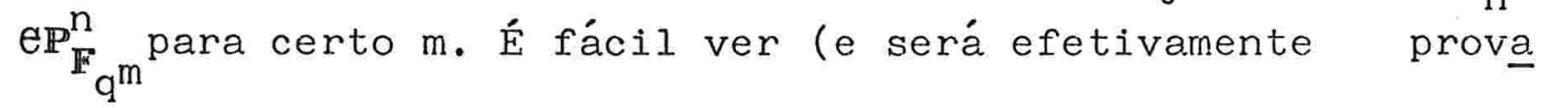
do no lema 1 do $\S 1$ do Capítulo IV) que $\mathrm{r} / \mathrm{m}$, o que implica que $r$ é o menor inteiro tal que $p \subset \mathbb{P}_{\mathbb{F}^{r}}^{n} \cdot \operatorname{Assim~} O_{\mathrm{P}}(\mathbb{C}) \subset$ $\mathbb{P}_{\mathrm{q}} \mathrm{r}(\mathbb{C})$, onde $\mathbb{F}_{\mathrm{q}} \mathrm{r}(\mathbb{C})$ é o corpo de funções da variedade C extendida a $\mathbb{F}_{q}$ r. E portanto temos um morfismo de corpos $\mathrm{O}_{\mathrm{P}}(\mathrm{C}) / \mathrm{M}_{\mathrm{P}}(\mathrm{C}) \rightarrow \mathrm{F}_{\mathrm{q}} \mathrm{r}$ dado pela avaliação em $\mathrm{P}$. Além disso, essa aplicação é $\mathbb{F}_{\mathrm{q}} \mathrm{r}-\mathrm{q}$ inear, e portanto é sobrejetora.

$$
\operatorname{Assim} \operatorname{dim}_{\mathbb{F}_{\mathrm{q}}} \mathrm{O}_{\mathrm{P}}(\mathrm{C}) / \mathrm{M}_{\mathrm{P}}(\mathrm{C})=\operatorname{dim}_{\mathbb{F}_{\mathrm{q}}} \mathbb{F}_{\mathrm{q}}^{\mathrm{r}}=\mathrm{r} \text {. }
$$




\section{CAPÍIIULO IIII}

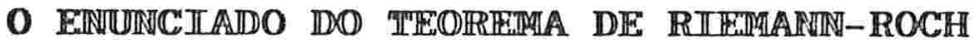

o teorema de Riemann-Roch é a ferramenta básica pa ra a prova da racionalidade da função zeta, e a originalidade de Stepanov e Bombieri foi sobretudo a utilização engenho sa desse teorema, obtendo assim uma prova "elementar" da $\mathrm{H} i$ pótese de Riemann para curvas. Trata-se de um teorema verdadeiramente fundamental e justamente celebrado.

Não faremos aqui uma prova desse teorema, pois pa ra fazê-lo de forma compreensiva necessitariamos de mais es paço do que dispomos, e além disso, se o teorema de RiemannRoch é essencial para o nosso trabalho, não o é a sua demons tração.

No livro de Fulton, Algebraic Curves, existe uma desmonstração que poderíamos chamar de "clássica" e que, en tre outros inconvenientes, considera apenas corpos algebrica mente fechadas e se utiliza de um modelo plano da curva pro jetiva. Isso, além de tornar a prova deselegante e complicada, acarretaria a necessidade de aumentar demasiadamente o capítulo II, tanto no concernente à geometria algébrica quan to à álgebra comutativa.

Já a prova de Chevalley, no seu livro clássico de funções algébricas, é relativamente mais curta e tem a vanta gem de considerar corpos arbitrários. Sua única desvantagem é ser incompreensível; pelo menos sem um trabalho complementar que coloque a sua definição de diferencial num contexto adequado. Seguindo de perto Chevaley, Max Deuring (Lectures on the theory of algebraic functions of one variable) repete - com um pouco mais de detalhes - a prova de Chevalley, po rém com a mesma definição de diferencial. 
Numa outra direção existem as provas de A.Weil (Ba sic Number Theory) e de Borel e Serre (Le théo-reme de Riemnn -Roch, Bull.Soc.Math. de France 86(1958), pp.97-136) que, ape sar de serem as mais curtas, se utilizam de uma maquinaria que não poderíamos desenvolver aqui - como cohomologia de feixes, teoria da dualidade, etc..., de modo que será mais conveniente aos nossos propósitos, simplesmente enunciar, cla ra e precisamente, o teorema de Riemann-Roch; o que faremos a seguir.

Vimos no capitulo II que se c é uma curva projetí va não singular definida sobre um corpo finito $\mathbb{F}_{q}$, então existe uma correspondência bijetora entre os pontos fecha dos de $\mathbb{C}$ e os anéis de valorização discreta de $\mathbb{F}_{q}$ (C) que con tém $F_{q}$, e que se $p$ é um ponto fechado, $|p|$ é igual à dimeñ são sobre $\mathbb{F}_{q}$ do corpo de restos da valorização associada $\bar{a}$ p.

Seja $D(\mathbf{C})$ o grupo abeliano livre gerado pelo con junto dos pontos fechados de C. Assim um elemento A de D(C) será chamado de divisor de c, e será escrito:

$$
A=\sum_{p} n_{p} p
$$

onde $n_{p}$ são inteiros quase sempre nulos, isto é nulos exceto para um número finito de pontos fechados. Definimos o grau de um divisor $A$ como $\operatorname{deg}(A)=\sum_{p} n_{p}|p|$, e é claro que: deg: $D(\mathbf{C})+z$ é um homomorfismo de grupos. Dizemos que o di visor $A=\sum_{p} n_{p} p$ é efetivo se $n_{p} \geq 0$ para todo $p$. Escrevemós $A \geq B$, onde ${ }^{p} B=\sum_{p} m_{p} p$, se $n_{p} \geq m_{p}$ para todo $p$, ou seja se
$A-B$ é efetivo. 
PROPOSICÃO I - Seja C uma curva projetiva não singular defí nida sobre $\mathbb{F}_{\text {q }}$. Se $f \in \mathbb{F}_{q}(\mathbf{C}), f \neq 0$, existe apenas um número finito de pontos fechados $p$, para os quais $v_{p}(f) \neq 0$, onde $v_{p}$ é a valorização associada à $p$.

Para a prova dessa proposição consultar, por exem plo, Deuring, op.cit., pg.24. Com essa proposição, se $f \in F_{q}(\mathbf{C}), f \neq 0$, podemos associar à. $f$ o divisor:

$$
(f)=\sum_{p} v_{p}(f) p
$$

Um divisor $A \in D(C)$ será chamado divisor principal se $A=(f)$ para algum $f \in \mathbb{F}_{q}(\mathbf{C})$. É claro que $\circ$ conjunto $\mathrm{D}_{\mathrm{p}}(\mathbf{C})$ dos divisores principais é um subgrupo de $\mathrm{D}(\mathbf{C})$, e $\quad$ grupo quociente, $D(\mathbf{C}) / D_{p}(\mathbf{C})$ é chamado o grupo das classes de divisores de C.

Demonstra-se que $\operatorname{deg}((f))=0$ (Deuring, pg 27) e portanto a aplicação $\operatorname{deg}: \mathrm{D}(\mathbf{C})+\mathbf{Z}$ fatoriza-se naturalmente à $\operatorname{deg}: \mathrm{D}(\mathbf{C}) / \mathrm{D}_{\mathrm{P}}(\mathbf{C})+\mathbf{Z}$.

junto:

$$
\text { Se } A=\sum_{p} n_{p} p \text { é um divisor de } \mathbb{C} \text {, definimos o con }
$$

$$
L(A)=\left\{f \in \mathbb{F}_{q}(\mathbf{C}):(f)+A \text { é efetivo }\right\}
$$

Assim $f \in \mathbb{q}_{\mathrm{q}}(\mathbf{C})$ está em $L(A)$ se $v_{p}(f) \geq-n_{p}$ ou se $f=0$. É claro que $L(A)$ é um $F_{q}$ - espaço vetorial, e denotaremos por $l(A)$ a sua dimensão.

PROPOSIÇÃO 2 - (a) se $A, B \in D(C)$ e $A \leq B$ então $L(A) \subset L(B)$ e $\operatorname{dim}_{F_{q}}(L(B) / L(A)) \leq \operatorname{deg}(B-A)$

(b) $L(O)=F_{q}, L(A)=0$ se $\operatorname{deg}(A)<0$ 
(c) $L(A)$ tem dimensão finita para todo $A$, e se $\operatorname{deg}(A) \geq 0$ en tão $I(A) \leq \operatorname{deg}(A)+1$

(d) Se $A-B=(f)$ então $l(A)=l(B)$

Para a demonstração dessa proposição

ver

Chevalley, (op.cit. pg 14, e pg 21).

Estamos agoram em condições de enunciar o teorema que é conhecido como:

TEOREMA DE RIEMANN. Seja C uma curva projetiva não singular sobre $\mathbb{F}_{\mathrm{q}}$. Existe uma constante $\mathrm{g}$ tal que $I(A) \geqslant \operatorname{deg}(A)+1-g$ para todos os divisores A. O menor g para a qual a desigualdade acima é verdadeira é chamado o gênero (genus) de C, e $\mathrm{g}$ é um inteiro não negativo. Além disso existe um inteiro $\mathrm{N}$ tal que para todos os divisores A de grau > N,

$$
I(A)=\operatorname{deg}(A)+1-g .
$$

Essa é a contribuição de Riemann ao chamado teore ma de Riemann-Roch. Para prova ver Chevalley, pg 2l-22.

TEOREMA DE RIEMANN-ROCH. Seja C uma curva projetiva não sin gular sobre um corpo finito $\mathbb{F}_{\mathrm{q}}$. Então existe um divisor $\mathrm{K}$ (que depende só de $\mathbf{c}$ ) tal que

$$
I(A)=\operatorname{deg}(A)+1-g+I(K-A)
$$

onde $\mathrm{A}$ é um divisor qualquer, $\mathrm{g}$ o gênero de $\mathbf{C}$.

OBSERVAÇÃO: pondo $A=0$ e $A=K$ na equação acima obtemos $I(K)=g$ e $\operatorname{deg}(K)=2 g-2$. Assim, se $\operatorname{deg}(A)>2 g-2$, $\operatorname{deg}(K-A)=2 g-2-\operatorname{deg}(A)<0$ donde $l(K-A)=0$, e assim, se $\operatorname{deg}(A)>2 g-2, I(A)=\operatorname{deg}(A)+1-g$. 
OBSERVAÇÃO. Se C/F $\mathbb{F}_{\mathrm{q}}$ é uma curva projetiva não singular sobre $\mathbb{F}_{q}$, f $\in \mathbb{F}_{q}(\mathbf{C})$, e $p$ um ponto fechado de $\mathbf{C}$, dizemos que $p \quad e ́$ um zero de $f$ se $v_{p}(f)>0$ e que $p$ é um polo de $f$ se $v_{p}(f)<0$. 


\section{CAPITULO IV}

\section{A HIPÓTEESE: DE RUEMUANIN PARA CURYAS SOBRE CORPOS FINITOS}

\section{$\$ 1$ - A Função ZEETIA đle uma Variedade $\mathbb{A}$ gébrica}

Para maior simplicidade expositiva, neste capitulo faremos algumas modificações na notação e na terminologia até aqui estabelecidas. Se $F_{q}$ é um corpo finito com q ele mentos, um ideal $\mathrm{x} \subset \mathbb{F}_{\mathrm{q}}\left[\mathrm{x}_{1}, \ldots, \mathrm{x}_{\mathrm{N}}\right]$ séra chamado de variedade afim, e um ideal homogêneo de $\mathbb{F}_{q}\left[x_{0}, x_{1}, \ldots, x_{N}\right]$ será châ mado de variedade projetiva. Escrevemos $\mathrm{X} / \mathbb{F}_{\mathrm{q}}$ para denotar in distintamente uma variedade afim ou projetiva sobre $\mathbb{F}_{q}$. Se $\mathrm{X} / \mathrm{F}_{\mathrm{q}}$ é uma variedade afim e $\mathrm{L} / \mathbb{F}_{\mathrm{q}}$ uma extensão de corpos, de finimos:

$$
\begin{aligned}
& X(L)=\left\{\left(x_{1}, x_{2}, \ldots, x_{n}\right) \in \mathbb{A}_{L}^{N}:\right. \\
& \left.: f\left(x_{1}, \ldots, x_{n}\right)=0, \quad \forall f \in X\right\}
\end{aligned}
$$

e de modo análogo definimos $X(L)$ no caso projetivo. Diremos que a variedade $X / \mathbb{F}_{q}$ é irredutivel se $X(L)$ o for, no sentido usual do capitulo II.

0 ideal nulo de $\mathrm{F}_{\mathrm{q}}\left[\mathrm{X}_{1}, \mathrm{x}_{2}, \ldots, \mathrm{X}_{\mathrm{N}}\right]$ será denotado $\mathbb{A}^{N}$ e o ideal nulo de $\mathbb{F}_{q}\left[\mathrm{X}_{0}, \mathrm{X}_{1}, \ldots . \mathrm{X}_{\mathrm{N}}\right]$ será denotado $\mathbb{P}^{N}$. Com a notação introduzida acima, temos $\mathbb{A}^{N}(L)=\mathbb{A}_{L}^{N}$ e $\mathbb{P}^{N}(L)=\mathbb{P}_{L}^{N}$. Se né um inteiro $\geq 1, x / \mathbb{F}_{\mathrm{q}}$ uma variedade sobre $\mathbb{F}_{\mathrm{q}}$ e $\mathrm{L} / \mathbb{F}_{\mathrm{q}}$ uma extensão finita de corpos, $|X(L)|$ denotará a cardinalidade de $X(L)$. Se $L=\mathbb{F}_{q}$ n temos claramente $\left|\mathbb{A}_{L}^{N}\right|=q^{n N}$ $\left|\mathbb{P}_{L}^{N}\right|=\frac{q^{n(N+1)}-1}{q^{n}-1}$ e, em particular se $x / \mathbb{F}_{q}$ é projetiva, , $|x(L)| \leq \frac{q^{n(N+1)}-1}{q^{n}-1}=1+q^{n}+\ldots+q^{n N}$ 
DEFINIÇÃO. Seja $X / F_{q}$ uma variedade sobre $F_{q}$. Definimos a sé rie formal na variável $t$ :

$$
z\left(x / \mathbb{F}_{q} ; t\right)=\exp \left(\sum_{n \geq 1}\left|x\left(\mathbb{F}_{q} n\right)\right| \frac{t^{n}}{n}\right)
$$

Essa expressão é chamada de função zeta da variedade $X / \mathbb{F}_{\mathrm{q}}$. Se $Q[t]]$ designar anel das séries formaj.s em $t$ com coeficientes racionais, é claro que $\mathrm{Z}\left(\mathrm{X} / \mathbb{F}_{\mathrm{q}} ; \mathrm{t}\right) \in 1+\mathrm{t} Q[[\mathrm{t}]]$. Se $\mathrm{X}$ for a variedade $\mathbb{A}^{N}$,

$$
\begin{gathered}
Z\left(\mathbb{A}^{N} ; t\right)=\exp \left(\sum_{n \geq 1}\left|\mathbb{A}^{N}\left(q^{n}\right)\right| \frac{t^{n}}{n}\right)= \\
=\exp \left(\sum_{n \geq 1} q^{n N} \frac{t^{n}}{n}\right)=\frac{1}{1-q^{N}}
\end{gathered}
$$

e se $\mathrm{X}$ for $\mathbb{P}^{N}$ teremos:

$$
\begin{gathered}
Z\left(\mathbb{P}^{N} ; t\right)=\exp \left(\sum_{n \geq 1}\left|\mathbb{P}^{N}\left(\mathbb{F}_{q^{n}} n\right)\right| \frac{t^{n}}{n}\right)=\exp \left(\sum_{n \geq 1} \frac{q^{n(N+1)}-1}{q^{n}-1} \frac{t^{n}}{n}\right) \\
=\exp \left(\sum_{n \geq 1} \sum_{i=0}^{N} \frac{\left(q^{i} t\right)^{n}}{n}\right)=\prod_{i=0}^{N} \exp \left(\sum_{n \geq 1} \frac{\left(q^{i} t\right)^{n}}{n}\right) \\
=\prod_{i=0}^{N} \frac{1}{1-q^{i} t}
\end{gathered}
$$

$\mathrm{SE} X / \mathbb{F}_{\mathrm{q}}$ é uma variedade arbitrária então $\mathrm{Z}\left(\mathrm{X} / \mathbb{F}_{\mathrm{q}} ; \mathrm{t}\right)$ define uma função holomorfa na variável complexa $t$, para $|t|<q^{-N}$. No caso afim isso decorre da (majoração) $\left|X\left(F_{q} n\right)\right| \leq q^{n N}$ e no projetivo, de $\left|X\left(\mathbb{F}_{q} n\right)\right| \leq 1+q^{n}+\ldots+q^{n N}$.

Se fizermos a mudnaça de variável $t=q^{-S}$ e escrevermos: 


$$
\zeta((X) ; s)=Z\left(X / F_{q} ; q^{-S}\right)
$$

segue das considerações acima que $\zeta(\mathrm{X} ; \mathrm{S})$ é uma série de Dirichlet convergente no semi-plano $\operatorname{Re}(\mathrm{s})>\mathrm{N}$.

Antes de continuar, relembramos a observação já feita, de que salvo menção contrária, as variedades consideradas serão sempre irredutíveis. Se $x / \mathbb{F}_{\mathrm{q}}$ é uma variedade projetiva não singular de dimensão $r$ definida sobre $\mathbb{F}_{q}$, te mos as "conjecturas de Weil":

(1) Existem $2 r+1$ famílias de inteiros algébricos $\left(\alpha_{j i}\right)_{1 \leq j \leq B_{i}}$ $0 \leq i \leq 2 r$ tais que, se para cada $i, P_{i}(t)=\underset{j=1}{\mathrm{~B}_{i}}\left(1-\alpha_{j i} t\right)$ então

$$
Z\left(X / \mathbb{F}_{q} ; t\right)=\frac{P_{1}(t) P_{3}(t) \ldots P_{2 r-1}(t)}{P_{0}(t) P_{2}(t) \ldots P_{2 r}(t)}
$$

e além disso $P_{0}(t)=1-t$ e $P_{2 r}(t)=1-q^{r} t$

(2) (Equação Funcional). Se pusermos $x=\sum_{i=0}^{2 r}(-1)^{i} B_{i}$ então:

$$
\mathrm{Z}\left(\mathrm{X} / \mathbb{F}_{\mathrm{q}} ; \frac{1}{\mathrm{q}^{\mathrm{r} t}}\right)= \pm \mathrm{q}^{\mathrm{rx} / 2} \mathrm{t}^{\mathrm{x}} \mathrm{Z}\left(\mathrm{x} / \mathbb{F}_{\mathrm{q}} ; \mathrm{t}\right)
$$

(3) (Hipótese de Riemann). Para todo par de índices $j, i$ tem- se

$$
\left|\alpha_{i j}\right|=q^{i / 2}
$$

(4) (Racionalidade dos "polinômios de Weil"). Cada um dos polinômios $P_{i}$ tem coeficientes inteiros racionais, de termo constante igual à 1 .

Se $\mathrm{X}$ for uma curva projetiva não singular definida 
sobre $\mathbb{F}_{\mathrm{q}}$, temos, em particular

$$
z\left(x / \mathbb{F}_{q} ; t\right)=\frac{P(t)}{(1-t)(1-q t)}
$$

onde $P(t)$ é um polinômio com coeficientes inteiros racionais de grau $2 \mathrm{~g}$ ( $\mathrm{g}$ é o gênero da curva), coeficiente dominante iglial a $\mathrm{q}^{\mathrm{g}}$ e termo constante 1 .

(2') (Equação Funcional). $P(t)$ satisfaz a equação funcional

$$
P(1 / q t)=q^{-g} t^{-2 g} P(t)
$$

(3') (Hipótese de Riemann). Os zeros de $P(t)$ têm módulo $q^{-1 / 2}$.

OBSERVAÇÕES (1): Decorre de (2) acima que a função zeta para curvas verifica:

$$
\mathrm{Z}\left(\mathrm{X} / \mathbb{F}_{\mathrm{q}} ; \mathrm{l} / \mathrm{qt}\right)=\mathrm{q}^{1-\mathrm{g}} \mathrm{t}^{2-2 \mathrm{~g}} \mathrm{z}\left(\mathrm{X} / \mathbb{F}_{\mathrm{q}} ; \mathrm{t}\right)
$$

(2) Se fizermos a mudança de variável $t=q^{-s}$, podemos reescrever ( $\left.3^{\prime}\right)$ acima, pondo que os zeros de $\zeta(x ; s)$ estão sobre a reta

$$
\operatorname{Re}(s)=\frac{1}{2}
$$

Ao longo do trabalho veremos que enquanto (1') e (2') são consequências quase diretas do teorema de Riemann-Roch, a Hipótese de Riemann para curvas, (3'), embora também decor ra desse teorema, é bem mais difícil de deduzir.

No capítulo II consideramos a ação natural de $\operatorname{Gal}\left(\overline{\mathbb{F}}_{\mathrm{q}} / \mathbf{F}_{\mathrm{q}}\right)$ sobre $X\left(\overline{\mathbb{F}}_{\mathrm{q}}\right)$ e chamamos de ponto fechado a uma ór 
bita dessa ação. Vimos também - pelas considerações que se seguem a proposição 6 do capítulo I - que todo ponto fecha do possui um número finito de elementos. Se p é um ponto fechado de grau $r$, e fácil ver que cada um dos elementos de p está em $\mathbb{A}_{\mathbb{F}_{q}}^{r}$ ou $\mathbb{P}_{\mathbb{F}_{q}}^{r}$, conforme $X$ seja afim ou projetiva. Assim, se ${ }^{q}{ }^{n}(X)$ designar o número de pontos fechados de $X\left(\overline{\mathbb{F}}_{q}\right)$ de grau $n, B_{n}(X)$ é finito.

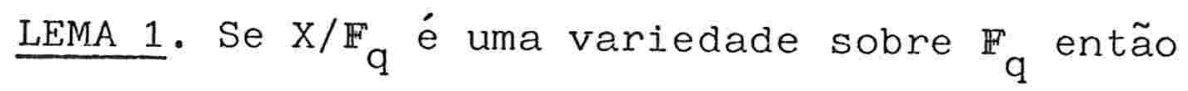

$$
\left|X\left(F_{\mathrm{q}} \mathrm{n}\right)\right|=\sum_{\mathrm{r} \mid \mathrm{n}} r \mathrm{~B}_{\mathrm{r}}(\mathrm{X})
$$

PROVA. Seja a $\in X\left(F_{q} n\right), a=\left(x_{1}, x_{2}, \ldots, x_{N}\right)$ no caso afim, ou $a=\left(x_{0}, x_{1}, \ldots, x_{N}\right)$ no caso projetivo, tal que sua órbita $\left\{a, a^{q}, a^{q^{2}}, \ldots, a^{q^{r-1}}\right\}$ tenha $r$ elementos. No caso afim, temos $x_{j} q^{r}=x_{j}, j=1,2, \ldots, N$. Se todos os $x_{j}$ forem nulos, então $r=1$ e $1 \mid n$. Se os $x_{j}$ não forem todos nulos então existe um certo $x_{j} \neq 0$ cujo período $\left(\right.$ em $\mathbb{F}_{q}^{*}$ ) é exatamente $q^{r}-1$, serão a órbita de a não poderia ter tamanho $r$, e assim $x_{j} \in \mathbb{F}_{q} r$, mas então $x_{j}$ é um gerador de $\mathbb{F}_{\mathrm{q}}^{*} \mathrm{r}$, donde $\mathbb{F}_{\mathrm{q}} \mathrm{r} \subset \mathbb{F}_{\mathrm{q}} \mathrm{n}$ e pela pro posição 2 do capítulo I, rln.

No caso projetivo, $x_{j}^{q^{r}}=\lambda x_{j}, j=0,1, \ldots, N$.

Se $x_{i}$ é um elemento não nulo da classe a,

e o resto é análogó.

$$
\begin{aligned}
& \text { então }\left(\frac{x_{j}}{x_{i}}\right)^{r}=\frac{x_{j}}{x_{i}}, j=0,1, \ldots, N \text {, donde } \frac{x_{j}}{x_{i}} \in \mathbb{F}_{q}^{r} \\
& \text { é análogó. }
\end{aligned}
$$

Agora, decompondo $X\left(\mathbb{F}_{\mathrm{q}} \mathrm{n}\right)$ como reunião disjunta de suas órbi tas e aplicando a definição de $B_{r}(X)$, obtemos:

$$
\left|X\left(F_{q} n\right)\right|=\sum_{r \leq n} r B_{r}(X)
$$


Mas acabamos de provar que se $X\left(\mathbb{F}_{q} n\right)$ possui uma órbita de tamanho $r$, então $r / n$. C.Q.D.

TEOREMA 1. Seja X/F $\mathbb{q}_{\mathrm{q}}$ uma variedade sobre $\mathbb{F}_{\mathrm{q}} \cdot$ Então

$$
\mathrm{Z}\left(\mathrm{x} / \mathbb{F}_{\mathrm{q}} ; t\right)=\mathrm{I}_{\mathrm{p}} \frac{1}{\left(1-\mathrm{t}^{|p|}\right)}
$$

onde o produto é tomado sobre todos os pontos fechados de $\mathrm{X}\left(\overline{\mathrm{F}}_{\mathrm{q}}\right)$.

PROVA: Tomando a derivada logarítmica de $\mathrm{Z}\left(\mathrm{X} / \mathbb{F}_{\mathrm{q}} ; \mathrm{t}\right)$ e aplican do o lema 1 obtemos:

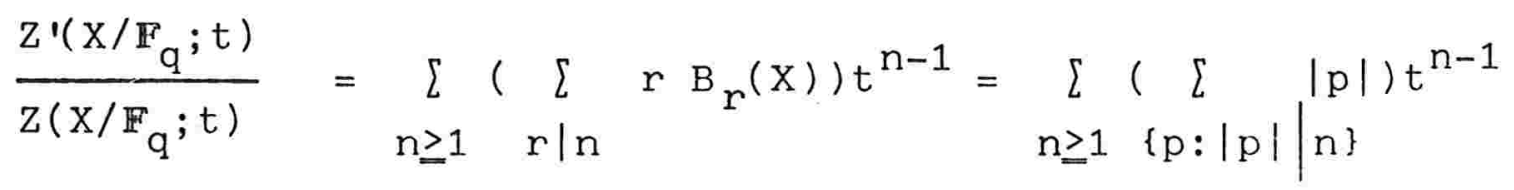

$$
\begin{aligned}
& =\sum_{n \geq 1} \sum|p| t^{m|p|-1}=\sum_{p} \frac{|p| t|p|-1}{\left(1-t^{|p|}\right)} \\
& =\sum_{p} \frac{\left[\left(1-t^{|p|}\right)^{-1}\right]^{\prime}}{\left[(1-t|p|)^{-1}\right]}=\left(\sum_{p} \log (1-t|p|)^{-1}\right)^{\prime} \\
& =\left[\log z\left(x / \mathbb{F}_{q} ; t\right)\right]^{\prime}=\left[\log \pi_{p}^{\pi} \frac{1}{\left(1-t^{|p|}\right)}\right]^{\prime}
\end{aligned}
$$

donde

$$
\mathrm{Z}\left(\mathrm{X} / \mathbb{F}_{\mathrm{q}} ; \mathrm{t}\right)=\mathrm{k} \cdot \mathrm{m}_{\mathrm{p}} \frac{1}{\left(1-\mathrm{t}^{|\mathrm{p}|}\right)}
$$


como $\mathrm{Z}\left(\mathrm{X} / \mathrm{F}_{\mathrm{q}} ; 0\right)=1$, temos $\mathrm{k}=1$.

$$
\text { C.Q.D. }
$$

Se $X / \mathbb{F}_{q}$ é uma variedade sobre $\mathbb{F}_{\mathrm{q}}$ e L/F $\mathbb{F}_{\mathrm{q}}$ é uma ex tensão de corpos, podemos pensar na variedade $\mathrm{X} \otimes \mathrm{L} / \mathrm{L}$ obtida por extensão de escalares, como fizemos no capítulo II.Relem bramos que $X \otimes L$ é o ideal gerado por $X$ em $L\left[X_{1}, X_{2}, \ldots, X_{N}\right]$ ou em $L\left[X_{0}, X_{1}, \ldots, X_{N}\right]$. É claro que, na nova notação,

$$
(X \otimes L)(E)=X(E) \text {, }
$$

para uma extensão E/L.

PROPOSIÇÃO 1. Seja d um inteiro $\geq 1 \mathrm{e} X / \mathbb{F}_{\mathrm{q}}$ uma variedade de finida sobre $\mathbb{F}_{\mathrm{q}} \cdot$ Então:

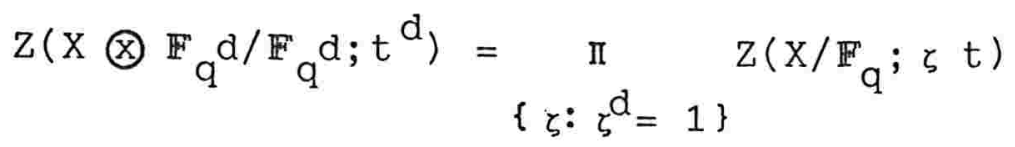

PROVA: Seja $\theta$ um gerador do grupo das raízes d-ésimas da unidade e $\mathrm{n}$ um inteiro $\geq 1$. Então

$$
\theta^{\mathrm{n}}\left(\sum_{\left\{\zeta: \zeta^{\mathrm{d}}=1\right\}} \zeta^{\mathrm{n}}\right)=\sum_{\zeta}\left(\begin{array}{ll}
\theta & )^{\mathrm{n}}=\sum_{\zeta} \zeta^{\mathrm{n}}
\end{array}\right.
$$

donde segue que

$$
\left(\theta^{n}-1\right)\left(\sum_{\zeta} \zeta^{n}\right)=0
$$

Assim, se d $X n, \theta^{n} \neq 1$ e portanto $\sum_{\zeta} \zeta^{n}=0$. 
Se $d \mid n, \quad \zeta^{n}=1$ e $\quad \sum \zeta^{n}=d$. Vamos aplicar essas observações à funçạ̃o:

$$
\begin{aligned}
& \pi \exp \left(\sum\left|x\left(\mathbb{F}_{\mathrm{q}}^{n}\right)\right| \frac{(\zeta t)^{n}}{n}\right)= \\
& \left\{\zeta \quad \zeta^{d}=1\right\} \quad n>1 \\
& =\exp \left(\sum_{\zeta} \sum_{n \geq 1}\left|x\left(\mathbb{F}_{q}^{n}\right)\right| \frac{(\zeta t)^{n}}{n}\right)
\end{aligned}
$$

A proposição estará provada se mostrarmos que

$$
\sum_{n \geq 1} \mid x\left(\mathbb{F}_{q} \text { nd }\right)\left|\frac{t^{n d}}{n}=\sum_{\zeta} \sum_{n \geq 1}\right| x\left(\mathbb{F}_{q}^{n}\right) \mid \frac{\zeta^{n} t^{n}}{n}
$$

Mas

$$
\begin{gathered}
\sum_{\zeta \geq 1}\left|x\left(\mathbb{F}_{q} n\right)\right| \frac{\zeta^{n} t^{n}}{n}=\sum_{n \geq 1}\left|x\left(\mathbb{F}_{q}^{n}\right)\right| \frac{t^{n}}{n}\left(\sum \zeta^{n}\right)= \\
\quad \sum_{m \geq 1}\left|x\left(\mathbb{F}_{q} m d\right)\right| \frac{t^{m d} \cdot d}{m d}
\end{gathered}
$$

C.Q.D. 


\section{$\$ 2$ - A Função ZEETA de Curvas Algébricas}

Neste parágrafo consideraremos sempre uma curva projetiva não singular $\mathbf{C} / \mathbb{F}_{\mathrm{q}}$ definida sobre $\mathbb{F}_{\mathrm{q}} \cdot 0$ teorema 1 nos permite escrever

$$
\begin{gathered}
\mathrm{z}\left(\mathbf{C} / \mathbb{F}_{q} ; t\right)=\prod_{\mathrm{p}} \frac{1}{1-\mathrm{t}^{|p|}}=\pi_{\mathrm{p}}\left(1+\mathrm{t}^{|p|}+\mathrm{t}^{2|p|}+\ldots\right) \\
=\sum_{n \geq 0} A_{n} t^{n}
\end{gathered}
$$

onde $A_{0}=1$ e $A_{n}$ é o número de divisores efetivos de grau $n$, para $n \geq 1$.

Se $E_{n}$ designa o conjunto dos divisores efetivos de grau $\mathrm{n}$ e $\mathrm{S}_{\mathrm{n}}$ é um conjunto de representantes das classes de divisores de grau $\mathrm{n}$, temos

$$
E_{n}=\bigcup_{A \in S_{n}}\left\{B: B \text { é efetivo, } B \equiv A\left(\bmod D_{P}(C)\right)\right\}
$$

ou seja,

$$
\begin{aligned}
E_{n}=\bigcup_{A \in S_{n}}\{A+(f): A+(f) \text { é efetivo }\}= \\
=\bigcup_{A \in S_{n}}\{A+(f): f \in L(A), f \neq 0\}
\end{aligned}
$$


Mas

$$
|\{A+(f): f \in L(A) ; f \neq 0\}|=|\{(f): f \in L(A), f \neq 0\}|
$$

e como $(f)=(g)$ se e só se $f=k g$ para algum $k \in \mathbb{F}_{q}^{*}$, então

$$
|\{(f): f \in L(A), f \neq 0\}|=\frac{|\{f: f \in L(A), f \neq 0\}|}{q-1}
$$

e

$$
\frac{|\{f: f \in L(A), f \neq 0\}|}{q-1}=\frac{q^{l(A)}-1}{q-1}
$$

donde

$$
A_{n}=\sum_{A \in S} \frac{q^{1(A)}-1}{q-1}
$$

e podemos escrever a função zeta como:

$$
(q-1) z(\mathbb{C} / \mathbb{F} ; t)=\sum_{n \geq 0} t^{n} \sum_{A \in S_{n}}\left(q^{l(A)}-1\right)
$$

Embora seja evidente, é bom observar que das con siderações acima decorre a finitude do número de classes de divisores de grau $n,\left|S_{n}\right|$. É importante notar também que $\left|S_{n}\right|=$ constante, para todo $n \geq 1$, pois se $A_{0}$ é um divisor de grau $\mathrm{n}_{\mathrm{o}}$, a aplicação $\mathrm{A}+\mathrm{D}_{\mathrm{p}}(\mathbf{C})+\mathrm{A}+\mathrm{A}_{\mathrm{o}}+\mathrm{D}_{\mathrm{p}}(\mathbf{C})$ é uma bijeção entre o conjunto das classes de divisores de grau $\mathrm{n}$ e o con junto das classes de divisores de grau $n+n_{0}$. No que segue, $\left|\mathrm{s}_{\mathrm{n}}\right|=\mathrm{h}, \mathrm{n} \geq 1$. 
Para obter mais informações sobre $h=\left|S_{n}\right|$, precí samos estudar a imagem do morfismo de grupos deg: $D(\mathbf{C})+\mathbf{Z}$. Seja dz o subgrupo de $\mathbf{Z}$ que é a imagem do homomorfismo deg. Então se $A_{o}$ é um divisor cujo grau é d e A é um divisor arbi trário, $\operatorname{deg}(A)=m \cdot \operatorname{deg}\left(A_{0}\right)$ para algum $m \in \mathbf{Z}$, donde

$$
d=m \cdot d \cdot c \cdot\{\operatorname{deg}(A): A \in D(C)\}
$$

ou seja, $d=\operatorname{m} . d . c\{|p|: p$ é ponto fechado\}. Vamos mostrar que $d=1$. Seja $n$ um inteiro $\geq 0$. Se d $\nmid n$ então não existem divisores de grau $n$, e se $d \mid n$, já sabemos que $\left|S_{n}\right|=h \quad$ e a função zeta fica:

$$
\begin{aligned}
& (q-1) z\left(\mathbf{c} / \mathbb{F}_{q} ; t\right)=\sum_{n \geq 0} t^{n d} \sum_{A_{n \in S}} q^{l(A)}-\sum_{n \geq 0} t^{n d_{h}} \\
& =\sum_{n \geq 0} t^{n d} \sum_{\text {AeS }} q^{l(A)}-\frac{h}{1-t^{d}}
\end{aligned}
$$

Se $\mathrm{K}$ é o divisor canônico (cf. Cap.III), já vimos que $\operatorname{deg}(K)=2 g-2$ e portanto d divide $2 g-2$ e a função zeta pode ser reescrita:

$$
\begin{gathered}
(q-1) \quad \mathbf{z}(\mathbf{C} / \mathbf{F} ; t)=\sum_{n=0}^{(2 g-2) / d} t^{n d} \sum_{A \in S_{n d}} q^{l(A)}+ \\
+\sum_{n>(2 g-2) / d} t^{n d} \sum_{A \in S_{n d}} q^{I(A)}-\frac{h}{1-t^{d}}
\end{gathered}
$$

Mas se $A \in S_{n d} e \operatorname{deg}(A)=n d>2 g-2$, sabemos que 


$$
I(A)=\operatorname{deg}(A)+1-g=n d+1-g,
$$

donde

$$
\begin{aligned}
& (2 g-2) / d \\
& (q-1) z\left(c / F_{q} ; t\right)=\sum_{n=0} t^{n d} \sum_{A \in S_{n d}} q^{1(A)}+ \\
& +\sum_{n>(2 g-2) / d} t^{n d} q^{n d+1-g} h-\frac{h}{1-t^{d}}
\end{aligned}
$$

ou seja:

$$
\begin{gathered}
(q-1) z\left(\mathbf{C} / \mathbb{F}_{q} ; t\right)=\sum_{n=0}^{(2 g-2) / d} t^{n d} \sum_{A \in S_{n d}} q^{I(A)}+ \\
+\frac{h q^{1-g}(t q)^{2 g-2+d}}{1-t^{d} q^{d}}-\frac{h}{1-t^{d}}
\end{gathered}
$$

PROPOSIĈ̃O 1: Seja p um ponto fechado de $\mathbb{C} / \mathbb{F}_{q}$ de grau $|p|$. Se $\ell|| p \mid$, podemos pensar em $p \otimes \mathbb{F}_{q}{ }^{l}$ como um ponto fechado de $\mathbf{C} \otimes \mathbb{F}_{\mathrm{q}}{ }^{1 / \mathbb{F}_{\mathrm{q}}}{ }^{l}$. Então $\left|\mathrm{p} \otimes \mathbb{F}_{\mathrm{q}}{ }^{1}\right|=|\mathrm{p}| / 1$.

PROVA: Seja $r=|p|$. Então p está contido em $\mathbf{C}\left(\mathbb{F}_{q} r\right)$. o esta bilizador de um elemento atp é o sugrupo $\mathrm{G}\left(\overline{\mathbb{F}}_{\mathrm{q}} / \mathbb{F}_{\mathrm{q}} \mathrm{r}\right)$ e portan to $r=\left|G\left(\bar{F}_{q} / \mathbb{F}_{q}\right) / G\left(\bar{F}_{q} / \mathbb{F}_{q} r\right)\right|$, pela toeria elementar das ações de grupos. Como $1 \mid r, \mathbb{F}_{q}{ }^{l} \subset \mathbb{F}_{\mathrm{q}} \mathrm{e}$ o estabilizador de $\mathrm{p} \otimes \mathbb{F}_{\mathrm{q}}{ }^{l}$ é

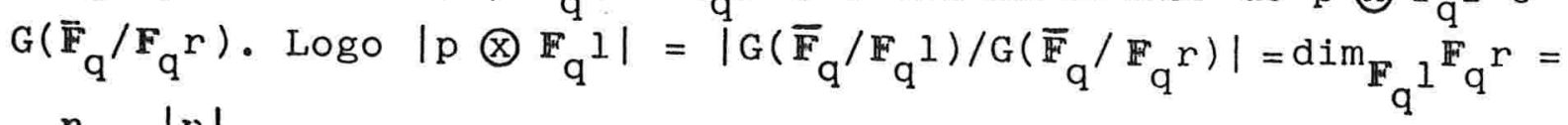
$=\frac{r}{I}=\frac{|p|}{l} \quad C \cdot Q \cdot D$.

A expressão (*) foi obtida para uma curva projetiva não singular $\mathbf{C} / \mathbf{F}_{\mathrm{q}}$, onde d era o gerador da imagem do homomor 
fismo deg:D(C) + Z. Façamos agora uma extensão de escalres pa ra $\mathbf{F}_{\mathrm{q}} \mathrm{d}$, considerando a curva $\mathbf{C} \times \mathbf{F}_{\mathrm{q}} \mathrm{d} / \mathbf{F}_{\mathrm{q}} \mathrm{d}$. Seja $\overline{\mathrm{d}} \circ$ gerador da imadem de $D\left(\mathbb{C} \otimes \mathbb{F}_{\mathrm{q}} \mathrm{d}\right)$ em $Z$. Pela proposição $1, \overline{\mathrm{d}}=1$. A foŕ mula (*) aplicada à $\mathbb{C} \otimes F_{q} d$ dá:

$$
\begin{aligned}
\left(q^{d}-1\right) \mathbb{Z}\left(\mathbf{C} \otimes \mathbf{F}_{q} d / \mathbb{F}_{q} d ; t\right)=\sum_{n=0}^{2 g-2} t^{n} \sum_{A \in S_{n}} q d l(A) \\
+\frac{\bar{h} \cdot q^{d(1-g)} t^{2 g-1} q^{d(2 g-1)}}{1-t q^{d}}-\frac{\bar{h}}{1-h}
\end{aligned}
$$

e no ponto $t^{d}$ obtemos:

$$
\begin{gathered}
\left(q^{d}-1\right) \mathbf{z}\left(\mathbf{c} \otimes \mathbb{F}_{q^{d} / \mathbb{F}_{q} d ; t} d\right)=\sum_{n=0}^{2 g-2} t^{n d} \sum_{A \in S_{n}} q^{d l(A)}+ \\
+\frac{\bar{h} \cdot q^{d(1-g)} t^{d(2 g-1)} q^{d(2 g-1)}}{1-t^{d} q^{d}}-\frac{\bar{h}}{1-t^{d}}
\end{gathered}
$$

Assim $Z\left(\mathbf{C} \otimes F_{q} d / F_{q} d ; t^{d}\right)$ possui um polo simples em $t=1$. Mas já vimos que

$$
z\left(\mathbf{C} \otimes \mathbb{F}_{q^{d} / \mathbb{F}} q^{\left.d ; t^{d}\right)=} \pi_{\left\{\zeta: \zeta^{d}=1\right\}}^{\pi} z\left(\mathbf{c} / \mathbb{F}_{q} ; \zeta t\right)\right.
$$

e portanto, da expressão (*), é imediato que

$$
\begin{gathered}
z\left(\mathbf{C} / \mathbf{F}_{\mathrm{q}} ; \zeta t\right)=\mathrm{z}\left(\mathbf{C} / \mathbb{F}_{\mathrm{q}} ; t\right) \mathrm{e} \\
z\left(\mathbf{C} \otimes \mathbb{F}_{\mathrm{q}} \mathrm{d} / \mathbb{F}_{\mathrm{q}} \mathrm{d} ; t^{\mathrm{d}}\right)=\left[\mathrm{z}\left(\mathbf{C} / \mathbb{F}_{\mathrm{q}} ; t\right)\right]^{d}
\end{gathered}
$$

Como $Z\left(\mathbf{C} / \mathbf{F}_{q} ; t\right)$ tem um polo simples em $t=1, Z\left(\mathbf{C} \otimes \mathbf{F}_{q} d / \mathbf{F}_{q} d ; t^{d}\right)$ 
teŕá um polo simples em $t=1$ se e só se $d=1$. Assim podemos reescrever a função zeta:

$$
\begin{gathered}
(q-1) z\left(\mathbf{c} / \mathbb{F}_{q} ; t\right)=\sum_{n=0}^{2 g-2} t^{n} \sum_{\operatorname{AES}_{n}} q^{l(A)}+ \\
+\frac{h \cdot q^{g} t^{2 g-1}}{1-q t}-\frac{h}{1-t}
\end{gathered}
$$

ou seja:

$$
\mathrm{Z}\left(\mathbf{C} / \mathbb{F}_{\mathrm{q}} ; t\right)=\frac{\mathrm{P}(\mathrm{t})}{(1-t)(1-\mathrm{q} t)}
$$

onde $P(t)$ é um polinômio de grau $2 g$, e $P(0)=1$. Vamos mos trar que o grau de $\mathrm{P}(\mathrm{t})$ é $2 \mathrm{~g}$. Para tanto vamos reescrever ex plicitamente a expressão geral da função zeta:

$$
(q-1) z\left(\mathbb{C} / \mathbb{F}_{q} ; t\right)=b_{0}+b_{1} t+\ldots+b_{2 g-2} t^{2 g-2}+\frac{h q^{g} t^{2 g-1}}{1-q t}-\frac{h}{1-t}
$$

onde

$$
b_{i}=\sum_{A \in S_{i}} q^{l(A)}
$$

PROPOSIÇÃO 2. Para $0 \leq i \leq 2 g-2, b_{i} q^{g-1-i}=b_{2 g-2-i}$.

PROVA: Já sabemos que se $\operatorname{deg}(A)=i$, então

$$
\operatorname{deg}(K-A)=2 g-2-i
$$

Assim, se $S_{i}=\left\{A_{1}, \ldots, A_{h}\right\}$ entã $\odot\left\{K-A_{1}, \ldots, K=A_{h}\right\} \dot{e} \quad$ um conjunto de representantes de classes de divisores de grau $2 \mathrm{~g}-2-\mathrm{i}$, isto é, $\left\{\mathrm{K}-\mathrm{A}_{1}, \ldots, \mathrm{K}-\mathrm{A}_{\mathrm{h}}\right\}=\mathrm{S}_{2 \mathrm{~g}-2-i} \cdot$ Assim, se 
$b_{i}=\sum_{j=1}^{h} q^{l\left(A_{j}\right)}$, então $b_{2 g-2-1}=\sum_{j=1}^{h} q^{l\left(K-A_{j}\right)}$

Mas pelo teorema de Riemann-Roch,

$$
\begin{gathered}
1\left(A_{j}\right)=i+1-g+l\left(K-A_{j}\right) \text {, donde temos, } \\
b_{2 g-2-i}=\sum_{j=1}^{h} q^{l\left(A_{j}\right)-i-1+g}=q^{g-1-i} b_{i}
\end{gathered}
$$

C.Q.D.

Se pusermos $t=0$ na expressão geral da função zeta, como $\mathbb{Z}\left(\mathbf{C} / \mathbb{F}_{\mathrm{q}} ; 0\right)=1$, segue

$$
\text { (q-1) } \mathbf{z}\left(\mathbf{C} / \mathbb{F}_{\mathrm{q}} ; 0\right)=\mathrm{b}_{\mathrm{o}}-\mathrm{h} \text {, donde } \mathrm{b}_{\mathrm{o}}=\mathrm{q}-1+\mathrm{h}
$$

e portanto $b_{2 g-2}=q^{g-1}(h+q-1)$. Decorre daí que $\circ$ termo in dependente de $\mathrm{P}(t)$ é 1 e o coeficiente de ordem $2 \mathrm{~g}$ é:

$$
\frac{q b_{2 g-2}-h q^{g}}{(q-1)}=\frac{q^{g}(h+q-1)-h q^{g}}{(q-1)}=q^{g}
$$

donde $\circ$ grau de $P(t)$ é $2 g$ e o coeficiente dominante é $q^{g}$.

$\underline{\text { PROPOSIÇÃO 3 }}$. (Equação Funcional) Se C/F $\mathbf{F}_{\mathrm{q}}$ é uma curva proje tiva não singular sobre $\mathbf{F}_{\mathrm{q}}$, então

$$
\mathbf{z}\left(\mathbf{C} / \mathbf{F}_{\mathrm{q}} ; \frac{1}{\mathrm{qt}}\right)=\mathrm{q}^{1-\mathrm{g}} \mathrm{t}^{2-2 \mathrm{~g}} \mathbf{z}\left(\mathbf{C} / \mathbf{F}_{\mathrm{q}} ; \mathrm{t}\right)
$$

PROVA: Substituindo $t$ por l/qt na expressão geral da função zeta obtemos: 


$$
\begin{aligned}
& (q-1) \quad \mathbf{z}\left(\mathbf{C} / \mathbb{F}_{q} ; \frac{1}{q t}\right)=b_{0}+\frac{b_{1}}{q t}+\ldots+\frac{b_{2 g-2}}{q^{2 g-2} t^{2 g-2}}+ \\
& +\frac{h q^{g}}{q^{2 g-1} t^{2 g-1}} \cdot \frac{1}{(1-1 / t)}-\frac{h}{1-1 / q t} \\
& =b_{0}+\frac{b_{1}}{q t}+\ldots+\frac{b_{2 g-2}}{q^{2 g-2} t^{2 g-2}}-\frac{q^{g_{h}}}{q^{2 g-1} t^{2 g-2}(1-t)}+\frac{q t h}{(1-q t)} \\
& =q^{1-g} t^{2-2 g}\left[b_{0} q^{g-1} t^{2 g-2}+b_{1} q^{g-2} t^{2 g-3}+\ldots+q^{1-g} b_{2 g-2}\right]- \\
& -\frac{q^{1-g} t^{2-2 g} h}{(1-t)}+\frac{q^{g} t^{2 g-1} h q^{1-q} t^{2-2 g}}{(1-q t)} \\
& =q^{1-g} t^{2-2 g}\left[\left(b_{2 g-2} t^{2 g-2}+\ldots+b_{0}\right)+\frac{h q^{g} t^{2 g-1}}{1-q t}-\frac{h}{1-t}\right] \\
& =q^{1-g} t^{2-2 g} \mathbf{z}\left(\mathbb{C} / \mathbb{F}_{q} ; t\right)
\end{aligned}
$$

Podemos escrever a função zeta como:

$$
\mathbb{Z}\left(\mathbb{C} / \mathbb{F}_{q} ; t\right)=\frac{i_{i=1}^{2 g}\left(1-\alpha_{i} t\right)}{(1-t)(1-q t)}
$$

onde os $\alpha_{i}$ são os inversos dos zeros de $P(t)$. 
A equação funcional para $\mathbf{Z}\left(\mathbb{C} / \mathbb{F}_{\mathrm{q}} ; t\right)$ implica imediata mente:

$$
P(t)=q^{g} t^{2 g} P\left(\frac{1}{q t}\right)
$$

e portanto se $1 / \alpha_{i}$ é raiz, $\alpha_{i} / q$ também será raiz de $P(t)$.

COROLÁRIO 1. Valem as igualdades:

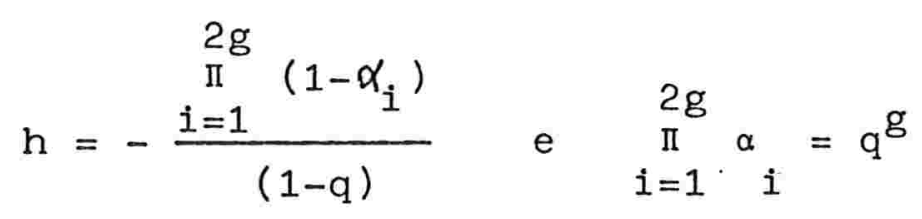

PROVA: A primeira igualdade exprime simplesmente o fato de que $-\mathrm{h}$ é o resíduo da função zeta no polo $t=1$. A segunda igualdade segue do fato de que o coeficiente dominante de $P(t)$ é $q^{g}$.

Precisamos mostrar que os $\alpha_{i}$ são inteiros algébri cos. Ora, provamos que $Z\left(\mathbb{C} / \mathbb{F}_{q} ; t\right)$ é uma função racional em $t$ com coeficientes inteiros. Sabemos que $Z\left(\mathbb{C} / \mathbb{F}_{q} ; 0\right)=1$ e que o desenvolvimento em série de potências $z\left(\mathbf{C} / \mathbb{F}_{q} ; t\right)=\sum_{n \geq 0} A_{n} t^{n}$ tem coeficientes inteiros.

Há um teorema, devido a Fatou (Acta Math., 30, pp 364-400) que diz o seguinte: se $F(t)$ é uma função racional com coeficientes em $Q$, se $F(O)=1$ e se $O$ desenvolvimento em série de Taylor de $F(t)$ possui todos os coeficientes intei ros, então os zeros e polos de $F(t)$ são inversos de inteiros algébricos. Isso mostra que os $\alpha_{i}$ são inteiros algébricos.

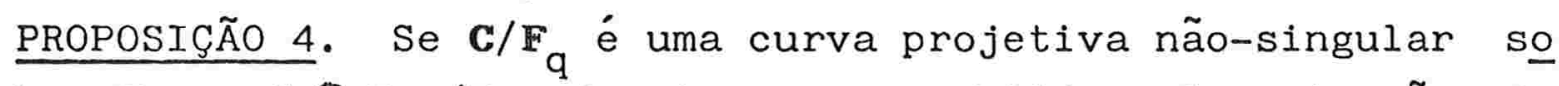
bre $\mathbf{F}_{\mathrm{q}}$ e $\quad \mathbf{C} \otimes \mathbb{F}_{\mathrm{q}} \mathrm{n} / \mathrm{F}_{\mathrm{q}} \mathrm{n}$ denota a curva obtida pela extensão de escalares à $F_{\mathrm{q}} \mathrm{n}$, então: 


$$
\mathbf{z}\left(\mathbf{C} \otimes \mathbb{F}_{q}{ }^{n / \mathbb{F}_{q}} \mathrm{n} ; \mathrm{t}\right)=\frac{\stackrel{2 g}{i=1}\left(1-\alpha_{i}^{n}\right)}{(1-t)\left(1-q^{n} t\right)}
$$

PROVA: Já vimos que a função zeta pode ser escrita como:

$$
Z\left(\mathbf{C} / \mathbb{F}_{q} ; t\right)=\frac{\prod_{i=1}^{2 g}\left(1-\alpha_{i} t\right)}{(1-t)(1-q t)}
$$

e além disso,

$$
\begin{aligned}
& \begin{array}{ll}
\pi_{\zeta=1}^{2 g} \\
& \left.\zeta \alpha_{i} \zeta t\right)
\end{array}
\end{aligned}
$$

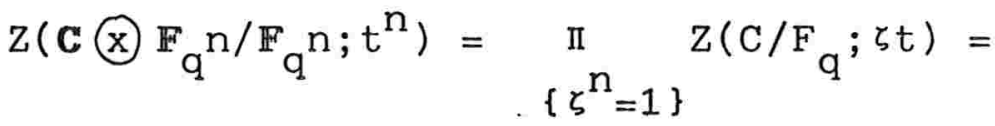

$$
\begin{aligned}
& \frac{\pi}{\zeta}(1-\zeta t)(1-q \zeta t)
\end{aligned}
$$

Mas $1-t^{n}=\underset{\zeta}{I I}(1-\zeta t)$, donde

$$
\mathrm{z}\left(\mathbf{c} \otimes \mathbb{F}_{q^{n}} \mathrm{~N} \mathbb{F}_{q}^{n} ; t^{n}\right)=\frac{\sum_{i=1}^{2 g}\left(1-\alpha_{i}^{n} t^{n}\right)}{\left(1-t^{n}\right)\left(1-q^{n} t^{n}\right)}
$$

e segue a proposição. $\quad$ C.Q.D.

COROLÁRIO 1. Se $z\left(\mathbf{C} / \mathbb{F}_{q} ; t\right)=\prod_{i=1}^{2 g}\left(1-\alpha_{i} t\right) /(1-t)(1-q t) \quad$ então

$$
\left|\mathbf{C}\left(\mathbb{F}_{q} \mathrm{n}\right)\right|=1+\mathrm{q}^{\mathrm{n}}-\sum_{i=1}^{2 \mathrm{~g}} \alpha_{i}^{\mathrm{n}}
$$

PROVA. Fazendo uma extensão de escalares à $\mathbb{F}_{\mathrm{q}} \mathrm{n}$, e aplicando a proposição 4 segue:

$$
\frac{\sum_{i=1}^{2 g}\left(1-\alpha_{i}^{n} t\right)}{(1-t)\left(1-q^{n} t\right)}=\exp \left(\sum_{k \geq 1}\left|\mathbf{C}\left(F_{q} n k\right)\right| \frac{t^{k}}{k}\right)=
$$




$$
=1+\left|\mathbf{C}\left(\mathbb{F}_{\mathrm{q}} \mathrm{n}\right)\right| \mathrm{t}+\ldots
$$

Por outro lado,

$$
\begin{aligned}
& { }_{i=1}^{2 g}\left(1-\alpha_{i}^{n} t\right)\left(1+t+t^{2}+\ldots\right)\left(1+q^{n} t+q^{2 n} t^{2}+\ldots\right)= \\
& =1+\left(1+q^{n}-\sum_{i=1}^{2 g} \alpha_{i}^{n}\right) t+\ldots
\end{aligned}
$$

igualando as duas séries obtemos:

$$
\left|\mathbb{C}\left(\mathbb{F}_{q} \mathrm{n}\right)\right|=1+\mathrm{q}^{\mathrm{n}}-\sum_{i=1}^{2 g} \alpha_{i}^{n}
$$

\section{\$ - PROVA DA HIPÓTESE DE RIERMANIN PARA CURVAS}

o trabalho preliminar já foi feito, e vamos agora provar a "hipótese de Riemann" para curvas, isto é, que

$$
\left|\alpha_{i}\right|=q^{1 / 2}
$$

ou equivalentemente, os zeros de $\zeta(\mathbf{C} ; S)$ estão sobre a reta $\operatorname{Re}(s)=\frac{1}{2}$.

Suponhamos por um isntante que essa "hipótese" se ja verdadeira, isto é, que $\left|\alpha_{i}\right|=q^{1 / 2}$. 
como

$$
\begin{gathered}
\left|\mathbf{C}\left(\mathbb{F}_{q^{n}}\right)\right|=1+q^{n}-\sum_{i=1}^{2 g} \alpha_{i}^{n} \text { temos } \\
|| \mathbf{C}\left(\mathbb{F}_{q^{n}}\right)\left|-1-q^{n}\right| \leq \sum_{i=1}^{2 g}\left|\alpha_{i}\right|^{n}=2 g q^{n / 2}
\end{gathered}
$$

No próximo lema provaremos que qualquer desigualda de do tipo acima é equivalente à "hipótese de Riemann".

LEMA 1. Suponhamos que exista um número $A>0$ tal que para todo $n \geqslant 1$,

$$
|| \mathbf{C}\left(\mathbb{F}^{n} q^{n)}\left|-1-q^{n}\right| \leq A q^{n / 2}\right.
$$

Então vale a "hipótese de Riemann".

PROVA: Por hipótese $\left|\sum_{i=1}^{2 g} \alpha_{i}^{n}\right| \leq A q^{n / 2}$

Consideremos a função:

$$
\sum_{i=1}^{2 g} \frac{1}{1-\alpha_{i} t}=\sum_{i=1}^{2 g} \sum_{n=0}^{\infty} \cdot\left(\alpha_{i} t\right)^{n}=\sum_{n=0}^{\infty}\left(\sum_{i=1}^{2 g} \alpha_{i}^{n}\right) t^{n}
$$

A série acima é majorada por $A \sum_{n=0}^{\infty} q^{n / 2}|t|^{n}$, e esta última converge para $|t|<\mathrm{q}^{-1 / 2}$. Logo, os polos $1 / \alpha_{i}$ da função $\sum_{i=1}^{2 g} \frac{1}{1-\alpha_{i} t}$ devem estar fora do
disco de raio $\mathrm{q}^{-1 / 2}$, isto é,

$$
q^{-1 / 2} \leq\left|\alpha_{i}\right|^{-1}, \text { ou seja, }\left|\alpha_{i}\right| \leq q^{1 / 2}
$$


Mas como $\prod_{i=1}^{2 g}\left|\alpha_{i}\right|=q^{g}=\left(q^{1 / 2}\right)^{2 g}$, segue necessariamente a igualdade $\left|\alpha_{i}\right|=q^{1 / 2}$

C.Q.D.

Assim, para estabelecermos a validade da "hipótese de Riemann" é suficiente provarmos que || $\mathbf{C}\left(\mathbb{F}_{\mathrm{q}} \mathrm{n}\right)\left|-1-\mathrm{q}^{\mathrm{n}}\right| \leq$ $A q^{n / 2}$ para todo $n \geq 1$ e para certo $A>0$. Na realidade, não precisamos provar essa desigualdade para todo $n \geq 1$ pois em virtude da proposição 4 do §2, a "hipótese de Riemann" para a curva $\mathbf{C}$ sobre $\mathbb{F}_{\mathrm{q}}$ é equivalente à "hipótese de Riemann" pa ra a curva extendida $\mathbf{C} \otimes \mathbb{F}_{\mathrm{q}} \mathrm{n}$ sobre $\mathbf{F}_{\mathrm{q}} \mathrm{n}$. Assim, podemos supor sem nenhuma perda de generalidade que $\mathrm{n}=1, \mathrm{q}>(\mathrm{g}+1)^{4}$, $q=p^{a}$ com a par. Ou seja, a constante A que encontraremos não depende do corpo de base.

o próximo teorema, cuja demonstração é devida à Stepanov-Bombier, dá conta da "metade" da "hipótese de Riemann". A outra "metade", que segue de um raciocínio de cahomologia goloisiana, seguirá adiante.

TEOREMA 1. Se $\mathbf{C} / \mathbb{F}_{\mathrm{q}}$ é uma curva projetiva não singular sobre $\mathrm{F}_{\mathrm{q}}$, de gênero $\mathrm{g}$, com $\mathrm{q}>(\mathrm{g}+1)^{4}, \mathrm{q}=\mathrm{p}^{\mathrm{a}}$, a par então:

$$
\left|\mathbf{C}\left(\mathbb{F}_{\mathrm{q}}\right)\right| \leq 1+q+(2 g+1) q^{1 / 2}
$$

PROVA: Evidentemente podemos supor $\mathbf{C}\left(\mathbb{F}_{\mathrm{q}}\right) \neq \varnothing$, pois caso contrário $\left|\mathbf{C}\left(\mathbb{F}_{q}\right)\right|=0$. Seja então $p \in \mathbf{C}\left(\mathbb{F}_{q}\right)$. p é um ponto fechado de grau 1, isto é, um ponto fixo de $\mathbf{C}\left(\overline{\mathbb{F}}_{q}\right)$ pela ação de $\operatorname{Gal}\left(\overline{\mathbf{F}}_{\mathrm{q}} / \mathrm{F}_{\mathrm{q}}\right)$. Seguindo Bombieri, consideraremos a curva C extendida ao fecho algébrico $\mathbb{F}_{q}$, i.e, $\mathbf{C} \otimes \overline{\mathbb{F}}_{q} / \overline{\mathbb{F}}_{q}\left(o u, \overline{\mathbf{C}} / \overline{\mathbb{F}}_{q}\right.$ ).

$$
\text { Seja } L_{m}=\left\{f \in \overline{\mathbf{F}}(\overline{\mathbf{C}}): v_{p}(f) \geq-m\right\} \text { e } I_{m} \text { a dimen }
$$
são de $L_{m}$ como $\mathbb{F}_{\mathrm{q}}$ - espaço vetorial. 
Temos $\overline{\mathrm{F}}_{\mathrm{q}}=\mathrm{L}_{\mathrm{o}} \subset \mathrm{L}_{1} \subset \ldots \subset \mathrm{L}_{\mathrm{m}} \subset \ldots$ e as propriedades abaixo:

(1) $1_{m} \geq m+1-g$ e, se $m>2 g-2$,

então $I_{m}=m+1-g$

(Isso ë simplesmente o teorema da Riemann, que enunciamos na pg.44).

(2) $1_{m+1} \leq 1+1_{m}$. (Do teorema de Riemann-Roch, $1_{m+1}-1_{m}=1+1(K-(m+1) p)-1(K-m p)$, e co mo $L(K-(m+1) p) \subseteq L(K-m p), \quad$ então $\left.1_{m+1}-1_{m} \leq 1\right)$.

(3) $\operatorname{Se} f(x) \in L_{m}$ então $f\left(x^{q}\right) \in L_{m q}$. (Isso simplesmente diz que se $f(X)$ tem em $p$ um polo de ordem $\leq m$, então $f\left(x^{q}\right)$ terá em $\mathrm{p}$ um pelo de ordem $\leq \mathrm{mq}$ ).

(4) Existe uma base $\left\{f_{1}, \ldots, f_{r}\right\}$ de $L_{m}$ tal que $v_{p}\left(f_{i}\right)<v_{p}\left(f_{i+1}\right)$ para $i=1,2, \ldots, r-1$, pois temos a filtração

$$
(0) \subset \overline{\mathbf{F}}_{\mathrm{q}}=1_{0} \subset \mathrm{L}_{1} \subset \cdots \subset \mathrm{L}_{\mathrm{m}} \mathrm{e}
$$

portanto

$$
\mathrm{L}_{\mathrm{m}} \simeq \bigoplus_{i=0}^{\mathrm{m}} \quad \mathrm{L}_{i} / \mathrm{L}_{i-1}
$$

Mas decorre da propriedade (2) que $\operatorname{dim} L_{i} / L_{i-1} \leq 1$, o que implica (4) pois pa ra cada $i$, quando for possível, tomamos um elemento de $L_{i}$ que não esteja em $L_{i-1}$. 
Sejam agora $\mathrm{n}, \mathrm{b}$ inteiros não negativos e sejam $s_{1}, \ldots, s_{r}$ elementos de $L_{n}$.

Consideremos a função auxiliar:

$$
F(x)=S_{1}^{p^{b}}(x) f_{1}\left(x^{q}\right)+\ldots+S_{n}^{p^{b}}(x) f_{n}\left(x^{q}\right)
$$

(5) $\quad$ Se $n p^{b}<q$, temos que $F(x)$ é identicamente nulo se e só se todos os $\mathrm{S}_{i}$ forem identicamente nulos.

De fato, suponhamos que $F(x)$ seja identicamente nulo e que $S_{h}(x)$ seja o primeiro $S_{i}$ não identicamente nulo. Tomando $v_{p}$ em ambos os lados da identidade:

$$
\begin{gathered}
s_{h}^{p^{b}}(x) f_{h}\left(x^{q}\right)=-S_{h+1}^{p^{b}}(x) f_{h+1}\left(x^{q}\right)-\ldots- \\
-S_{r}^{p^{b}}(x) f_{r}\left(x^{q}\right)
\end{gathered}
$$

obtemos,

$$
\begin{array}{r}
p^{b} v_{p}\left(S_{h}\right)+q v_{p}\left(f_{h}\right) \geq \min _{i>h}\left(p^{b} v_{p}\left(S_{i}\right)+\right. \\
\left.+q v_{p}\left(f_{i}\right)\right) \geq-p^{b} n+q v_{p}\left(f_{h+1}\right)
\end{array}
$$

e portanto

$$
\begin{aligned}
& p^{b} v_{p}\left(S_{h}\right) \geq-p^{b} n+q\left(v_{p}\left(f_{h+1}\right)-v_{p}\left(f_{h}\right)\right) \\
& \text { ou seja, por (4), } \\
& \qquad p^{b} v_{p}\left(S_{h}\right) \geq-p^{b} n+q>0
\end{aligned}
$$


donde segue que $\mathrm{S}_{\mathrm{h}}$ se anula em $\mathrm{p}$, e portanto é uma função sem polos com pelo menos um ze ro, ou seja, $v_{p}\left(S_{h}\right)>0$ e $v_{q}\left(S_{h}\right) \geq 0$ para todo $\mathrm{q} \neq \mathrm{p}$.

Como $\sum_{p} v_{p}\left(s_{h}\right)=0$, segue que $s_{h} \equiv 0$.

(6) Se $m, n>2 g-2$ e se $(n+1-g)(m+1-g)>$ $\mathrm{p}^{\mathrm{b}} \mathrm{n}+\mathrm{m}+1-\mathrm{g}$ então podemos escolher os $\mathrm{S}_{i}$ não todos identicamente nulos tal que a função

$s_{1}^{p^{b}}(x) f_{1}(x)+\cdots+s_{r}^{p^{b}}(x) f_{r}(x)$

seja identicamente nula.

De fato, essa função é regular fora de $p$ e tem em $\mathrm{p}$ um polo cuja ordem $\dot{e} \leq \mathrm{p}^{\mathrm{b}} \mathrm{n}+\mathrm{m}$.

Logo, por (1), o conjunto dessas funções for ma um $\overline{\mathbb{F}}_{\mathrm{q}}$-espaço vetorial de dimensão $\leq \mathrm{p}^{\mathrm{b}} \overline{\mathrm{n}}+$ $+m+1-g$.

Como cada $\mathrm{S}_{\mathrm{i}}$ varia num espaço de dimensão $\mathrm{n}+1-\mathrm{g}$ e como, $\mathrm{r}=\mathrm{m}+1-\mathrm{g}$ (pois $m>2 g-2)$, e por hipótese $(n+l-g)(m+1-g)>$ $\mathrm{p}^{\mathrm{b}} \mathrm{n}+\mathrm{m}+1-\mathrm{g}$ a aplicação canônica

$$
\mathrm{L}_{\mathrm{n}}^{\mathrm{p}^{\mathrm{b}}} \underset{\overline{\mathbf{F}}_{\mathrm{q}}}{(\mathrm{X})} \mathrm{L}_{\mathrm{m}} \rightarrow L\left(\left(\mathrm{p}^{\mathrm{b}} \mathrm{n+m}\right) \mathrm{p}\right)
$$

não pode ser injetora; o que prova (6). (Observe que $\operatorname{dim} L_{n}=\operatorname{dim} L_{n}^{p b}$ )

Assim, em vista de (5) e (6), obtemos que, se $m, n>2 g-2, n p^{b}<q$, 
$(\mathrm{n}+1-\mathrm{g})(\mathrm{m}+1-\mathrm{g})>\mathrm{np} \mathrm{b}^{\mathrm{b}}+\mathrm{m}+1-\mathrm{g}$, podemos construir uma função auxiliar

$$
F(x)=s_{1}^{p^{b}}(x) f_{1}\left(x^{q}\right)+\ldots+s_{r}^{p^{b}}(x) f_{r}\left(x^{q}\right)
$$

não identicamente nula e tal que se

a $\in \mathbb{C}\left(\mathbb{F}_{\mathrm{q}}\right)$ a $\neq \mathrm{p}$, então $F(\mathrm{a})=0$ (note que $\left.a^{q}=a\right)$.

Ou seja, F se anula em todos os pontos de $\mathbf{C}\left(\mathbb{F}_{\mathrm{q}}\right)$ exceto em $\mathrm{p}$. Como $\mathrm{p}^{\mathrm{b}}<\mathrm{q}$, por constru ção, vemos que $F(x)$ é uma potência $p^{b}$-ésima, isto é, $F(x)=G(x)^{p^{b}}$ para certa $G(x)$ $\in \overline{\mathbb{F}}_{\mathrm{q}}(\overline{\mathbf{C}})$ e assim cada zero de $F(x)$ tem multi pljcidade pelo menos $\mathrm{p}^{\mathrm{b}}$. Logo $F(x)$ tem pelo menos $p^{b}\left(\left|\mathbb{C}\left(\mathbb{F}_{q}\right)\right|-1\right)$ zeros. Logo

$$
\begin{aligned}
\left(\left|\mathbf{C}\left(\mathbb{F}_{q}\right)\right|-1\right) & \leq \underset{p^{b}}{\frac{1}{p}} \#(\text { zeros de } F)= \\
& =\frac{1}{p^{b}} \#(\text { polos de } F) \leq \\
& \leqslant \frac{1}{p^{b}}\left(p^{b} n+m q\right)
\end{aligned}
$$

pois $F$ é resular fora de $\mathrm{p}$ e a ordem do 10 em $p$ não pode exceder $p^{b} n+m q$.

Resumindo, até agora vimos que se $\mathrm{m}, \mathrm{n}>2 \mathrm{~g}-2, n \mathrm{p}^{\mathrm{b}}<\mathrm{q}$ e $(\mathrm{n}+1-\mathrm{g})(\mathrm{m}+1-\mathrm{g})>$ $n p^{b}+m+1-g$, 
então

$$
\left|\mathbf{C}\left(\mathbf{F}_{\mathrm{q}}\right)\right| \leq 1+\mathrm{n}+\frac{\mathrm{mq}}{\mathrm{p}}
$$

Tomando $\mathrm{p}^{\mathrm{b}}=\mathrm{q}^{1 / 2}, \mathrm{n}=\mathrm{q}^{1 / 2}-1, \mathrm{~m}=\mathrm{q}^{1 / 2}+2 \mathrm{~g} \quad \mathrm{e}$ lembrando que, por hipótese, $q>(g+1)^{4}$

(a) $\mathrm{n}=\mathrm{q}^{1 / 2}-1>(\mathrm{g}+1)^{2}-1=\mathrm{g}^{2}+2 \mathrm{~g}>2 \mathrm{~g}-2$

(b) $\mathrm{m}=\mathrm{q}^{1 / 2}+2 \mathrm{~g}>2 \mathrm{~g}-2$

(c) $(n+1-g)(m+1-g)=\left(p^{b}-g\right)\left(p^{b}+2 g+1-g\right)=$

$$
\begin{aligned}
& =\left(p^{b}-g\right)\left(p^{b}+g+1\right)=p^{b} p^{b}+p^{b} g+p^{b}-g p^{b}-g^{2}-g \\
& =p^{b} p^{b}+p^{b}-g-g^{2}>p^{b} p^{b}+g^{2}+2 g+1-g-g^{2}= \\
& =p^{b} p^{b}+1+g=n p^{b}+m+1-g
\end{aligned}
$$

(d) Como $\left(\mathrm{q}^{1 / 2}-1\right) \mathrm{q}^{1 / 2}<\mathrm{q}, \mathrm{np} \mathrm{p}^{\mathrm{b}}<\mathrm{q}$.

Assim, todas as condições estão verificadas e portanto

$$
\begin{aligned}
\left|\mathbf{C}\left(\mathbb{F}_{\mathrm{q}}\right)\right| & \leq 1+\left(q^{1 / 2}-1\right)+\frac{\left(q^{1 / 2}+2 g\right)}{q^{1 / 2}} \\
\text { ou seja: } & \begin{aligned}
\left|\mathbf{C}\left(\mathbf{F}_{\mathrm{q}}\right)\right| & \leq 1+q^{1 / 2}-1+q+2 g q^{1 / 2} \\
& \leq 1+q+(2 g+1) q^{1 / 2}
\end{aligned}
\end{aligned}
$$


Resta agora provar a outra "metade" da hipótese de Riemann, pois o argumento acima não nos dá uma limitação in ferior para $\left|\mathbf{C}\left(\mathbf{F}_{\mathrm{q}}\right)\right|$.

Por exemplo, se $\left|\mathbf{C}\left(\mathbb{F}^{r} \mathrm{q}\right)\right|=1+\mathrm{q}^{r}-\dot{\alpha}_{1}^{r}-\alpha_{2}^{r}$ $\alpha_{1}=\mathrm{q}, \alpha_{2}=1$, então $\alpha_{1} \alpha_{2}=\mathrm{q},\left|\mathbf{C}\left(\mathbb{F}_{\mathrm{q}} \mathrm{r}\right)\right|$ é sempre zero, mas $e^{1}$ falso que $\left|\alpha_{i}\right|=q^{1 / 2}$. A idéia básica para provar o que falta, é fazer um raciocínio de contagem sobre uma família de corpos conjugados, de uma extensão fixa de um corpo dado. A parte abstrata do raciocínio é o seguinte lema de contagem do número de pontos fixos da ação de um grupo finito num conjun to dado.

Seja G um grupo finito, $H$ um subgrupo de $G, N_{G}(H)$ o normalizador de $\mathrm{H}$ em $\mathrm{G}$ e $\sigma$ um elemento fixo de $\mathrm{N}_{\mathrm{G}}(\mathrm{H})$. Seja $X$ um conjunto onde $G$ age $e x \in X$. A órbita de $x$ pela ação de $G$ será denotada $O_{G}(x)$ e o conjunto das órbitas da ação de $G$ em $X$ será escrito $G \backslash X$.

Como $\mathrm{H}$ é subgrupo de $\mathrm{G}, \mathrm{H}$ age em $\mathrm{X}$ pela restrição da ação de $G$, e como $\sigma \in \mathrm{N}_{\mathrm{G}}(\mathrm{H})$, $\sigma$ induz uma aplicação $\hat{\sigma}$ : $\mathrm{H} \backslash \mathrm{X}+\mathrm{H} \backslash \mathrm{X}$ dada por $\hat{\sigma} \cdot O_{\mathrm{H}}(\mathrm{x})=\mathrm{O}_{\mathrm{H}}(\sigma \mathrm{x})$. É imediato que $\hat{\sigma}$ está bem definida. Se $g \in G, F i x(g)$ denota o conjunto dos $x \in X$ tais que $g x=x e$, como sempre, $|F i x(g)|$ denota a cardinalida de de $\operatorname{Fix}(g)$.

PROPOSIÇÃO 1. Com as hipóteses e notações acima vale:

$$
\sum_{h \in H}|\operatorname{Fix}(\sigma h)|=|H| \cdot|\operatorname{Fix}(\hat{\sigma})|
$$

PROVA: Seja $A=\bigcup_{\text {hEH }} F i x(\sigma h)$ e a seguinte relação de equivalên cia em $A: x \sim y \quad \operatorname{se}_{H}(x)=O_{H}(y)$. É imediato que se $x \in A$, $\mathrm{O}_{\mathrm{H}}(\mathrm{x}) \subset \mathrm{A}$ e $\mathrm{O}_{\mathrm{H}}(\mathrm{x}) \in \mathrm{Fix}(\hat{\sigma})$, donde segue que $\mathrm{A} / \sim=\mathrm{Fix}(\hat{\sigma})$. Que remos computar $\sum_{h \in H}|F i x(h)|$. Se $x \in A, x$ é contado $\left|H_{x}\right|$ vezes 


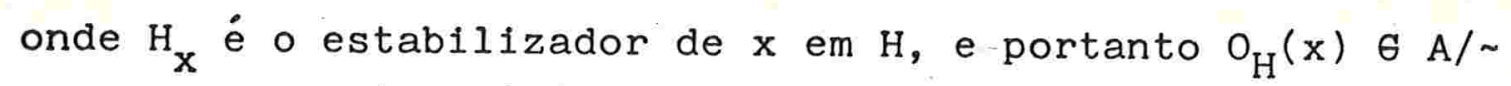
contribui com $\left|\mathrm{H}: \mathrm{H}_{\mathrm{x}}\right| \cdot\left|\mathrm{H}_{\mathrm{x}}\right|$ vezes, pois os estabilizadores de elementos da mesma órbita são conjugados.

Assim, cada elemento de $\mathrm{A} / \sim$ é contado em $\sum_{h \in H}|f i x(\sigma h)|,|H|$ vezes, donde

$$
|\operatorname{Fix}(\hat{\sigma})|=|\mathrm{A} / \sim|=\frac{1}{|H|} \sum_{h \in H}|\operatorname{Fix}(\sigma h)|
$$

Retornando ao problema original, seja $\mathrm{k}$ um corpo finito com $q$ elementos, $K / \mathrm{k}$ um corpo de funções algébricas de uma variável sobre $\mathrm{k}$ e L/K uma extensão de Galois finita. Po demos considerar $K$ como o corpo de funções racionais de uma curva $\mathrm{C} / \mathrm{k}$. Designamos por $\mathrm{V}_{1}(\mathrm{~K} / \mathrm{k})$ o conjunto das valorizações discretas de $K$ que se anulam em $k$ e cujo corpo de restos tem grau 1 sobre $k$.

Já vimos no capítulo II que se $\mathrm{c} / \mathrm{k}$ é uma curva pró jetiva não singular sobre $k$, que existe uma correspondência bijetora entre os pontos fechados de grau 1 de $\mathrm{C}$ e os ele mentos de $V_{1}(\mathrm{~K} / \mathrm{k})$.

Vamos agora provar o teorema 1, abaixo, que nos permitirá completar a prova da "hipótese de Riemann". A pro va que fizemos foi baseada em notas de um seminário da Karl otto stöhr.

TEOREMA 2. Seja $\mathrm{k}$ um corpo finito com q elementos, $\mathrm{K} / \mathrm{k}$ um corpo de funções algébricas de uma variável e L/K uma exten são uma extensão de Galois finita. Além disso suponhamos que $\mathrm{k}$ seja algebricamente fechado em L. Então existem [L:K] cor pos de funções algébricas de uma variável, $\mathrm{L}_{\mathrm{t}} / \mathrm{k}$, $t \in \mathrm{Gal}(\mathrm{L} / \mathrm{K})$ verificando: 
(a)

$$
L_{i d}=L \quad \ldots
$$

(b)

$$
\mathrm{L}_{\mathrm{t}} \supset \mathrm{K} \text { e }\left[\mathrm{L}_{\mathrm{t}}: \mathrm{K}\right]=[\mathrm{L}: \mathrm{K}]
$$
(c) $L_{t} \cdot \bar{k}=L_{0} \bar{k}$, onde $\bar{k}$ é um fecho algé brico de $k$

(d)

$$
\underset{t \in G(L / K)}{\sum \cdot\left|V_{1}\left(L_{t} / K\right)\right|=[L: K] \cdot\left|V_{1}(K / K)\right|}
$$

PROVA. Seja $\mathrm{m}=[\mathrm{L}: \mathrm{K}]$ e $\mathrm{k}_{\mathrm{m}} / \mathrm{k}$ uma extensão de grau $\mathrm{m}$. Como $\mathrm{L} \cap \mathrm{k}_{\mathrm{m}}=\mathrm{k}, \mathrm{G}\left(\mathrm{k}_{\mathrm{m}} / \mathrm{k}\right) \simeq \mathrm{G}\left(\mathrm{Kk}_{\mathrm{m}} / \mathrm{K}\right) \simeq \mathrm{G}\left(\mathrm{Lk}_{\mathrm{m}} / \mathrm{L}\right), \mathrm{e}$

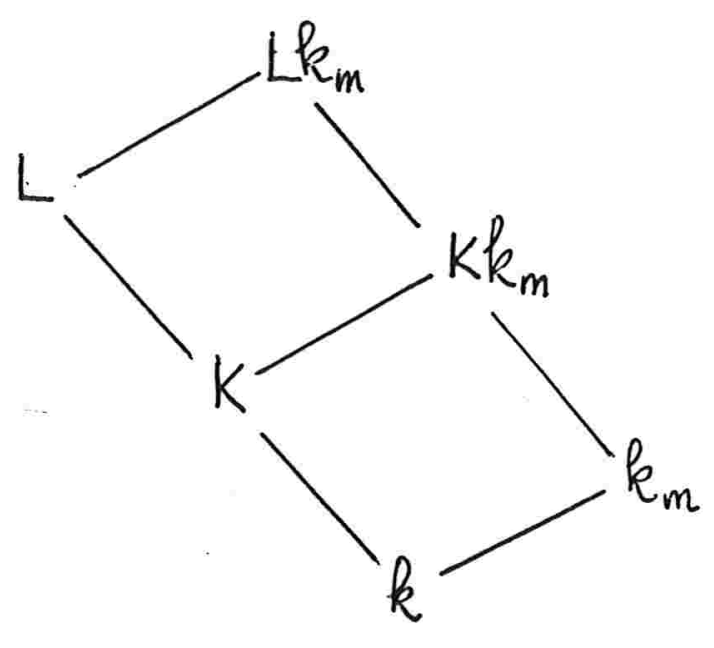

como $\mathrm{L} \cap \mathrm{Kk}_{\mathrm{m}}=\mathrm{K}$, então $\mathrm{G}(\mathrm{L} / \mathrm{K}) \simeq \mathrm{G}\left(\mathrm{Lk}_{\mathrm{m}} / \mathrm{Kk}_{\mathrm{m}}\right)$. Vimos no capítu lo I que $G(\mathrm{~km} / \mathrm{k})$ é cíclico e gerado pelo automorfismo de Frobenius $\mathrm{S}: \mathrm{a} \longmapsto \mathrm{a}^{\mathrm{q}}$. Seja $\sigma \circ$ automorfismo induzido por $\mathrm{S}$ em $G\left(L_{m} / L\right)$. Se $t \in G(L / K)$, seja $\tau$ o automorfismo induzido por $t$ em $G\left(L k_{m} / K_{m}\right)$. É claro que or é um automorfismo de Lkm sobre $\mathrm{k}$ e que $\sigma \tau=\tau \sigma$. Como $\circ$ período de $\sigma$ é $\mathrm{m}$ e $\circ \tau$ de divide $m$ se $f$ for $o$ período de $\sigma \tau$ então $f$ divide $m$. Mas se $x \in k m,(\sigma \tau)^{f}(x)=S^{f}(x)=x$ donde $m$ divide $f$, e portanto $m=f$, ou seja, a ordem de $\sigma \tau \dot{e}$ precisamente $m$.

$$
\text { Seja } L_{t}=\left\{a \in L k_{m}:(\sigma \tau) a=a\right\} \text { corpo fixo }
$$
pelo grupo gerado por $\sigma \tau$. Pela toeria de Galois a exten 
são $L_{m} / L_{t}$ é de Galois finita com grupo de Galois $\langle\sigma \tau\rangle$, ou seja $\left|\mathrm{Lk}_{\mathrm{m}}: \mathrm{L}_{\mathrm{t}}\right|=\mathrm{m}$.

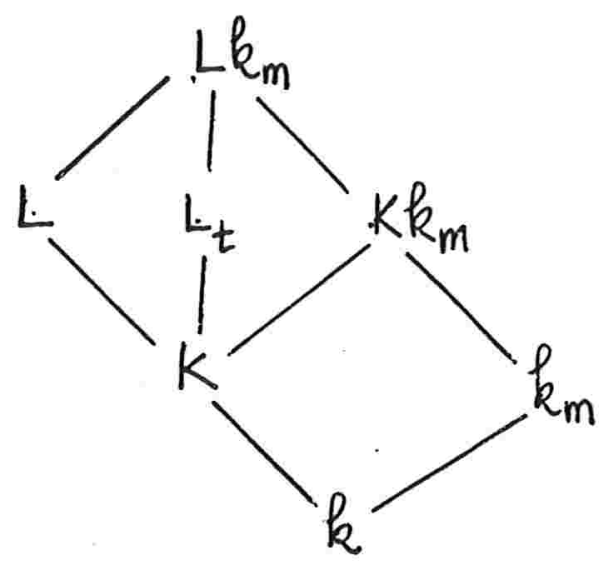

Decorre daí que $\mathrm{L}_{t} / \mathrm{k}$ é um corpo de funções algébrí cas de uma variável sobre $\mathrm{k}$. Além disso, $\mathrm{L}_{t} \mathrm{k}_{\mathrm{m}}=\mathrm{Lk}_{\mathrm{m}}$ pois có mo $L_{t} \subset L_{m}, L_{t} k_{m} \cap L_{m}$ e além disso:

$$
\mathrm{L}_{\mathrm{t}} \cap \mathrm{k}_{\mathrm{m}}=\left\{a \in \mathrm{k}_{\mathrm{m}}:(\sigma \tau) \mathrm{a}=\mathrm{a}\right\}=\left\{a \in \mathrm{k}_{\mathrm{m}}: \mathrm{S}(\mathrm{a})=\mathrm{a}\right\}=\mathrm{k}
$$

donde

$$
\left.\mid \mathrm{Lk}_{\mathrm{m}}: \mathrm{L}_{\mathrm{t}}\right]=\mathrm{m} \quad \mathrm{e} \operatorname{assim} \mathrm{L}_{\mathrm{t}} \mathrm{k}_{\mathrm{m}}=\mathrm{Lk}_{\mathrm{m}} \cdot
$$$$
\text { Note-se que } L_{t} \bar{k}=\left(L_{t} k_{m}\right) \bar{k}=\left(L_{m}\right) \bar{k}=L \bar{k} \text {. }
$$

Como $\mathrm{L} \cap \mathrm{Kk}_{\mathrm{m}}=\mathrm{K}$, temos da teoria de Galois que $\mathrm{G}\left(\mathrm{Lk}_{\mathrm{m}} / \mathrm{K}\right) \simeq \mathrm{G}(\mathrm{L} / \mathrm{K}) \mathrm{X} \mathrm{G}\left(\mathrm{Kk}_{\mathrm{m}} / \mathrm{K}\right)$, e portanto podemos pensar em $G(L / K)$ como subgrupo de $G\left(L k_{m} / K\right)$.

Vamos agora nos utilizar dos resultados acerca de valorização para compararmos certos cardinais de conjuntos de valorizações. Consideremos a seguinte situação: 


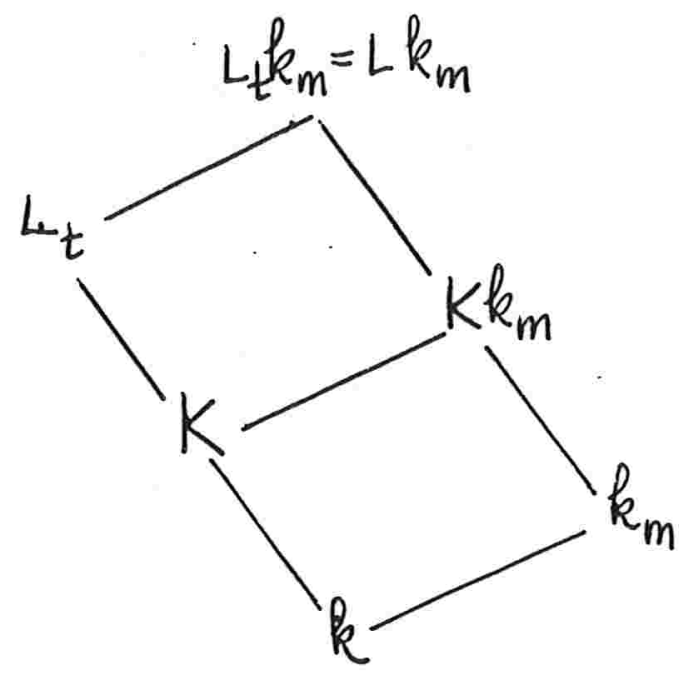

onde já vimos que $G\left(k_{m} / k\right) \simeq G\left(L_{t} k_{m} / L_{t}\right)$.

0 grupo $\mathrm{G}(\mathrm{Lkm} / \mathrm{K})$ age naturalmente em $\mathrm{V}\left(\mathrm{L} \mathrm{k}_{\mathrm{m}} / \mathrm{k}_{\mathrm{m}}\right)$ - o conjunto das valorizações discretas de Lk triviais em $k_{m} \cdot A$ ação é dada por $\psi_{\bullet} v+v \Psi^{-1}$. Como $G\left(L_{t} k_{m} / L_{t}\right)$ é subgru po de $G\left(L k_{m} / K\right)$, podemos pensar na ação desse subgrupo por restrição da ação de $G\left(L k_{m} / K\right)$.

Se $v \in V\left(L_{t} k_{m} / k_{m}\right)$, depois do lema $5, \S 2$, Cap. I, podemos considerar a restrição usual de funções para obtermos uma valorização $\left.v\right|_{L_{t}} \in V\left(L_{t} / k\right)$ ou seja, temos uma aplica-
ção:

$$
\text { Rest: } V\left(L_{t} k_{m} / k_{m}\right) \longrightarrow V\left(L_{t} / k\right)
$$

dada pela restrição usual de funções. Pelo teorema 1, § 2, Cap. I, rest é uma aplicação sobrejetora.

Já vimos também (teor.2, §2, Cap.I) que se $v \in V\left(L_{t} / k\right), \operatorname{Rest}^{-1}(v)$ é uma órbita da ação de $H_{t}=G\left(L_{t} k_{m} / L_{t}\right)$ em $V\left(L k_{m} / k_{m}\right)$. Assim existe um correspondên 
cia bijetora entre $V\left(L_{t} / k\right)$ e as órbitas $H_{t} \backslash V\left(L_{t} k_{m} / k_{m}\right)$.

Vamos agora provar que há uma correspondência bije tora entre $\mathrm{V}_{1}\left(\mathrm{~L}_{\mathrm{t}} / \mathrm{k}_{\mathrm{m}}\right)$ e Fix $\left(\mathrm{H}_{\mathrm{t}}\right)$ onde $\mathrm{Fix}\left(\mathrm{H}_{\mathrm{t}}\right)=\mathrm{v} \in \mathrm{V}_{1}\left(\mathrm{~L}_{\mathrm{t}} \mathrm{k}_{\mathrm{m}} / \mathrm{k}_{\mathrm{m}}\right)$ : $: \operatorname{vo\rho }^{-1}=\mathrm{v}, \forall \rho \in \mathrm{H}_{\mathrm{t}}$.

Seja então $v \in V\left(L_{t} / k\right)$ e $L_{t}(v) \circ$ seu corpo de restos $\left(L_{t}(v)=A_{v} / M_{v}\right)$. Seja $d(v)$ a sua dimensão como k-e $\underline{s}$ paço vetorial. Pela prop.1, §2, Cap.I, d(v) é finito. Se w $\in \mathrm{V}\left(\mathrm{L}_{\mathrm{t}} \mathrm{k}_{\mathrm{m}} / \mathrm{k}_{\mathrm{m}}\right)$ é uma extensão de $\mathrm{v}$ temos: o diagrama:

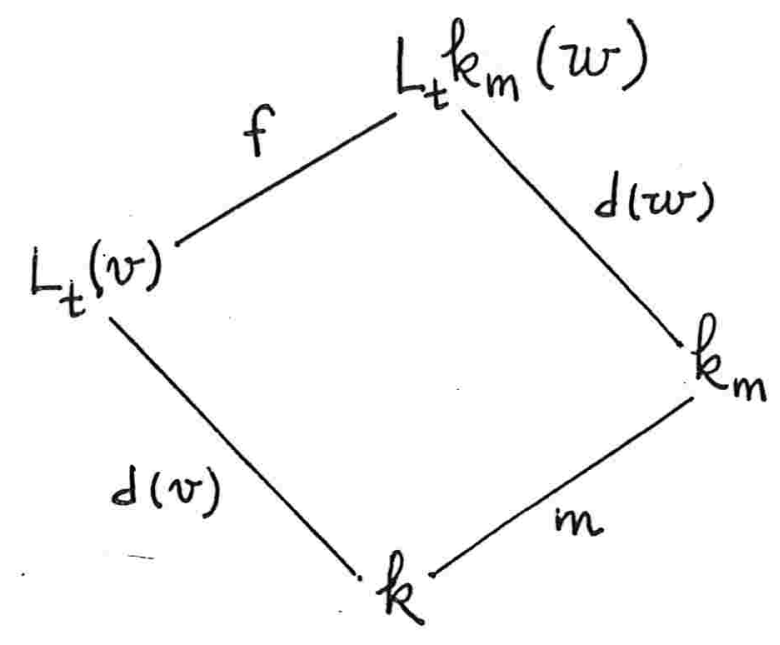

Se $g=\left|O_{H_{t}}(w)\right|$, isto é, é o número de extensões de $v \grave{a} L_{t} k_{m}$, pelo teórema 3 , §2, Cap.I, sabemos que e.f.g $=m$, donde $d(v) f=d(w) f g$, pois $e=1$ (Lema $5, \quad \S 2$, Cap.I), ou seja, $d(v)=g d(w)$.

Logo se $w \in F i x\left(H_{t}\right)$, sua órbita tem tamanho 1, don de $\left.\quad w\right|_{L_{t}}=v$ tem $d\left(\left.w\right|_{L_{t}}\right)=1$.

Reciprocamente, se $v \in v_{1}\left(L_{t} / k\right)$ e w extende $v$, en tão $1=g d(w)$, donde $g=1$ e $d(w)=1$. 
Assim:

$$
\begin{aligned}
& \mathrm{V}_{1}\left(\mathrm{~L}_{\mathrm{t}} / \mathrm{k}\right)=\left\{\left.\mathrm{w}\right|_{L_{t}}: \mathrm{w} \in \operatorname{Fix}\left(\mathrm{H}_{\mathrm{t}}\right)\right\} \\
& \text { Como } \mathrm{H}_{\mathrm{t}}=\langle\sigma \tau\rangle \text {, elaro que } \\
& \left|\mathrm{V}_{1}\left(\mathrm{~L}_{\mathrm{t}} / \mathrm{k}\right)\right|=\left|\mathrm{Fix}\left(\mathrm{H}_{\mathrm{t}}\right)\right|=|\mathrm{Fix}(\sigma \tau)|
\end{aligned}
$$

Pondo $\mathrm{G}=\mathrm{G}\left(\mathrm{Lk}_{\mathrm{m}} / \mathrm{K}\right), \mathrm{H}=\mathrm{G}(\mathrm{L} / \mathrm{K})$ e $\mathrm{X}=\mathrm{V}_{1}\left(\mathrm{Lk}_{\mathrm{m}} / \mathrm{k}_{\mathrm{m}}\right)$, como $\sigma \mathrm{t}=$ to para todo $t \in G(L / K), \sigma \in\left(N_{G}(H)\right.$ e pela proposição 1 des te $\S$,

$$
\begin{gathered}
\frac{1}{[L: K]} \sum_{t \in G(L / K)}\left|V_{1}\left(L_{t} / k\right)\right|=\frac{1}{|H|} \sum_{t \in H}|F i x(\sigma t)|= \\
=|\operatorname{Fix}(\hat{\sigma})|
\end{gathered}
$$

onde $\hat{o}$ é a aplicação induzida nas órbitas de $\mathrm{G}(\mathrm{L} / \mathrm{K})$ em $\mathrm{V}_{1}\left(\mathrm{Lk}_{\mathrm{m}} / \mathrm{k}_{\mathrm{m}}\right)$.

$$
\begin{aligned}
& \text { Resta mostrar que }|\mathrm{Fix}(\hat{\sigma})|=\left|\mathrm{V}_{1}(\mathrm{~K} / \mathrm{k})\right|: \\
& \hat{\sigma}: \frac{\mathrm{V}_{1}\left(\mathrm{Lk}_{\mathrm{m}} / \mathrm{k}_{\mathrm{m}}\right)}{\mathrm{H}} \longrightarrow \frac{\mathrm{V}_{1}\left(\mathrm{Lk}_{\mathrm{m}} / \mathrm{k}_{\mathrm{m}}\right)}{\mathrm{H}}
\end{aligned}
$$

$\dot{\mathrm{e}}$ dada por $\mathrm{O}_{\mathrm{H}}(\mathrm{v}) \longmapsto \mathrm{O}_{\mathrm{H}}\left(\mathrm{vo \sigma}^{-1}\right)$

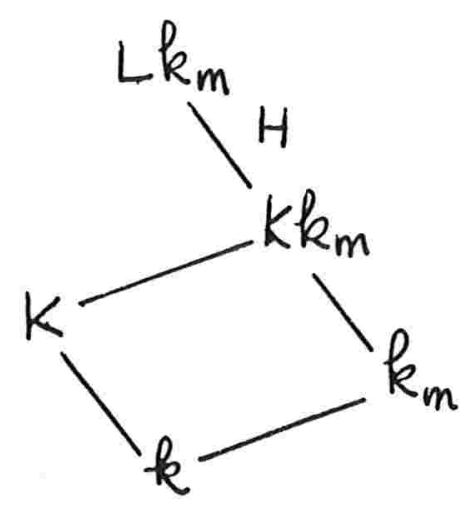

.78. 
Como $\mathrm{H}=\mathrm{G}(\mathrm{L} / \mathrm{K}) \simeq \mathrm{G}\left(\mathrm{Lk}_{\mathrm{m}} / \mathrm{Kk}_{\mathrm{m}}\right)$, temos na mesma situa ção da pg. 77, ou seja,

$$
|\operatorname{fix}(\hat{\sigma})|=\left|V_{1}(K / k)\right|
$$

Isso prova (d),

$$
\text { C.Q.D. }
$$

Observe-se que se $K=k(X)$ for o corpo de funções racionais (isto é, $\mathrm{K}$ é o corpo de funções da variedade pro jetiva $\mathbb{P}_{\mathbb{P}}^{1}$ ) então o número de pontos fechados de grau 1 , é precisamente

$$
\frac{q^{2}-1}{q-1}=q+1
$$

e portanto

$$
\sum_{\operatorname{t\in G}(L / k(x))}\left|V_{1}\left(L_{t} / K\right)\right|=[L: k(x)](q+1)
$$

É importante observar também que se $\mathbf{C} / \mathbb{F}_{\mathrm{q}}$ é uma curva projetịva não singular sobre $\mathbb{F}_{\mathrm{q}}$ e $\mathrm{K}=\mathbb{F}_{\mathrm{q}}\left(\mathbb{C}^{\prime}\right)$ é o corpo de funções racionais em $\mathbf{C}$, então é possivel encontrar um elemento $\theta$ transcendente sobre $\mathbb{F}_{\mathrm{q}}$ tal que a extensão $\mathrm{K} / \mathbb{F}_{\mathrm{q}}(\theta)$ seja separável (cf.Iyanaga, Theory of numbers, p.34). Assim, podemos tomar uma extensão $L / F_{q}(\theta)$ que seja de Galois finita e que contenha $K$, por exemplo $L=K^{\text {nor }}$, o fecho normal de $K$ sobre $\mathbb{F}_{\mathrm{q}}(\theta)$. 0 que não se pode garantir a priori é que $\mathbb{F}_{\mathrm{q}}$ seja algebricamente fechado em L. Para contornar esse pro blema, seja $k^{\prime}$ o fecho algébrico de $\mathbb{F}_{q}$ em L.

Como $\mathrm{L} / \mathbf{F}_{\mathrm{q}}$ é uma extensão finitamente gerada e $\mathrm{L} \supset \mathrm{k}^{\prime} \supset \mathbf{F}_{\mathrm{q}}, \mathrm{k}^{\prime} / \mathbf{F}_{\mathrm{q}}$ será finitamente gerada, e como é algébrí ca, decorre que $\mathrm{k}^{\prime} / \mathrm{F}_{\mathrm{q}}$ é finita. Assim podemos fazer uma ex 
tensão de escalares $C \otimes k^{\prime} / k^{\prime}$ e provar a hipótese de Riemann sobre $k^{\prime}$.

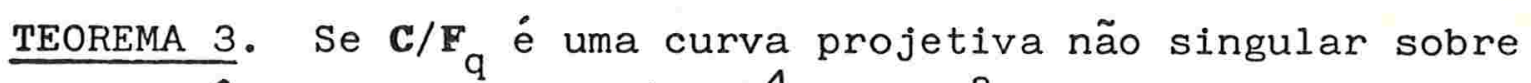
$\mathbf{F}_{\mathrm{q}}$, de gênero $\mathrm{g}$, com $\mathrm{q}>(\mathrm{g}+1)^{4}, \mathrm{q}=\mathrm{p}^{\mathrm{a}}$, a par, $\mathrm{K}=\mathbf{F}_{\mathrm{q}}(\mathbf{C})$ o corpo de funções racionais de $\mathbf{C}$ e $\mathrm{L} / \mathrm{K}$ uma extensão de Galois finita com $\mathbf{F}_{\mathrm{q}}$ algebricamente fechado em $\mathrm{L}$ então existe $\mathrm{A}>0$ tal que

$$
|| \mathbf{C}\left(\mathbf{F}_{\mathrm{q}}\right)|-1-\mathrm{q}| \leq \mathrm{A} \mathrm{q}^{1 / 2}
$$

PROVA: O teorema 1 deste parágrafo nos deu

$$
\left|\mathbf{C}\left(\mathbf{F}_{\mathrm{q}}\right)\right|-1-\mathrm{q} \leq(2 \mathrm{~g}+1) \mathrm{q}^{1 / 2}
$$

Aplicando esse mesmo teorema à familia de corpos de funções algébricas de uma variável $\mathrm{L}_{\mathrm{t}} / \mathbb{F}_{\mathrm{q}}$ obtemos:

$$
\left|v_{1}\left(L_{t} / F_{q}\right)\right| \leq(q+1)+\left(2 g_{L}+1\right) q^{1 / 2}
$$

onde $g_{L}$ è o gênero da curva associada à extensão $L / F_{q}$. As sim, se $S \in G\left(L / \mathbb{F}_{\mathrm{q}}(\theta)\right)$ temos:

$$
\sum_{\substack{\operatorname{tGG}\left(L / F_{q}(\theta)\right) \\ t \neq S}}\left|\mathrm{~V}_{1}\left(L_{t} / \mathbb{F}_{q}\right)\right| \leq\left(\left[L: \mathbb{F}_{q}(\theta)\right]-1\right)\left[(q+1)+\left(2 g_{L}+1\right) q^{1 / 2}\right]
$$

e

$$
\left|V_{1}\left(L_{S} / F_{q}\right)\right|=\left[L: \mathbb{F}_{q}(\theta)\right](q+1)-\sum_{t \neq S}\left|V_{1}\left(L_{t} / F_{q}\right)\right|
$$

Pondo $\left[L: F_{q}(\theta)\right]=n$ vem: 


$$
\begin{aligned}
\left|v_{1}\left(L_{S} / F_{q}\right)\right| & \geq n(q+1)-(n-1)\left[(q+1)+\left(2 g_{L}+1\right) q^{1 / 2}\right] \\
& \geq(q+1)-(n-1)\left(2 g_{L}+1\right) q^{1 / 2}
\end{aligned}
$$

donde

$$
\sum_{\operatorname{S\in G}(L / K)}\left|V_{1}\left(L_{S} / F_{q}\right)\right| \geq[L: K]\left[(q+1)-(n-1)\left(2 g_{L}+1\right) q^{1 / 2}\right]
$$

e portanto

$$
\left|v_{1}\left(K / \mathbb{F}_{q}\right)\right| \geq(q+1)-(n-1)\left(2 g_{L}+1\right) q^{1 / 2}
$$

tomando $A=\max \left\{(2 g+1),(n-1)\left(2 g_{L}+1\right)\right\}$

segue que

$$
|| \mathbf{C}\left(\mathbb{F}_{\mathrm{q}}\right)|-1-\mathrm{q}| \leq A \mathrm{q}^{1 / 2}
$$

C.Q.D. 


\section{BIBLIOGRAFIA}

[1]: E.Artin. Collected Papers. Springer Verlag

[2]: Atiyah e Macdonald. Introduction to Commutative Algebra. Adolison - Wesley (1969)

[3] : E.Bombieri. Counting Points on Curves over finite fields. Séminaire Bcurbaki 1973 n 430

[4]: W.Fulton. Algebraic Curves. W.A. Benjamin, Inc. 1969

[5]: R.Hartshorne. Algebraic Geometry. Springer-Verlag G.M.T.Vol.52. 